\title{
Optimality conditions for fractional differential inclusions with nonsingular Mittag-Leffler kernel
}

\author{
G.M. Bahaa ${ }^{1,2^{*}}$ and Adnane Hamiaz
}

\section{"Correspondence:}

Bahaa_gm@yahoo.com

'Department of Mathematics,

Faculty of Science, Taibah University,

Al-Madinah Al-Munawarah, Saudi

Arabia

2Present address: Department of

Mathematics and Computer

Sciences, Faculty of Science, Beni-Suef University, Beni-Suef, Egypt

\begin{abstract}
In this paper, by using the Dubovitskii-Milyutin theorem, we consider a differential inclusions problem with fractional-time derivative with nonsingular Mittag-Leffler kernel in Hilbert spaces. The Atangana-Baleanu fractional derivative of order $\alpha$ in the sense of Caputo with respect to time $t$, is considered. Existence and uniqueness of solution are proved by means of the Lions-Stampacchia theorem. The existence of solution is obtained for all values of the fractional parameter $\alpha \in(0,1)$. Moreover, by applying control theory to the fractional differential inclusions problem, we obtain an optimality system which has also a unique solution. The controllability of the fractional Dirichlet problem is studied. Some examples are analyzed in detail.

MSC: 46C05; 49J20; 93C20

Keywords: Fractional optimal control problems; Variational inequalities; Fractional differential systems; Cauchy problems; Existence and uniqueness of solutions; Riemann-Liouville sense; Caputo derivative; Atangana-Baleanu fractional derivative; Mittag-Leffler kernel; Dubovitskii-Milyutin theorem
\end{abstract}

\section{Introduction}

Fractional calculus was proposed independently by Newton and Leibniz. The theory of a non-integer order operators (integral and differential) was initiated in 1695. Ever since that time, several definitions of integral and differential operators have been suggested: Riemann-Liouville derivative and integral, Caputo derivative, Hadamard integral, Erdélyi-Kober fractional derivative and integral, Grunwald-Letnikov derivative, left and right Chen integrals.

In (2015), Caputo and Fabrizio [30] proposed a new definition of fractional derivatives:

$$
{ }^{\mathrm{CF}} D_{a+}^{\alpha} f(x)=\frac{B(\alpha)}{1-\alpha} \frac{\mathrm{d}}{\mathrm{d} t} \int_{a}^{x} \exp \left[\frac{-\alpha}{1-\alpha}(x-y)\right] f(y) \mathrm{d} y
$$

valid for $0<\alpha<1$, with $B(\alpha)$ being a normalization function satisfying $B(0)=B(1)=1$. The basic challenge they were addressing was whether it is possible to construct another type of fractional operator which has nonsingular kernel and which can better describe in some cases the dynamics of nonlocal phenomena. The Caputo-Fabrizio definition has already

(c) The Author(s) 2018. This article is distributed under the terms of the Creative Commons Attribution 4.0 International License (http://creativecommons.org/licenses/by/4.0/), which permits unrestricted use, distribution, and reproduction in any medium, provided you give appropriate credit to the original author(s) and the source, provide a link to the Creative Commons license, and indicate if changes were made. 
found applications in areas such as diffusion modeling [39] and mass-spring-damper systems [8].

However, some issues were pointed out against both derivatives, including one in the Caputo sense and one in the Riemann-Liouville sense. As Sheikh [48] pointed out, the CF fractional derivative as the kernel in integral was nonsingular but was still nonlocal. Some researchers also concluded that the operator was not a derivative with fractional order but a filter with fractional parameter. The fractional parameter can then be viewed as a filter regulator. To overcome the above drawbacks, Yang et al. [52] proposed a new fractional derivative involving the normalized sinc function without singular kernel.

Atangana and Baleanu introduced a new operator with fractional order based upon the generalized Mittag-Leffler function (see [1, 2, 10, 19-34, 40-45, 51] and the references therein). Their operators have all the benefits of that of the CF derivative in addition to the kernel being nonlocal and nonsingular. The nonlocality of the kernel gives better description of the memory within the structure with different scale.

Here we shall mostly be considering a more recently developed definition for fractional differintegrals due to [10]. This new type of calculus addresses the same underlying challenge as that of Caputo and Fabrizio, but it uses a kernel which is nonlocal as well as nonsingular, namely the Mittag-Leffler function:

$$
\begin{aligned}
& { }^{\mathrm{ABR}} D_{a+}^{\alpha} f(t)=\frac{B(\alpha)}{1-\alpha} \frac{\mathrm{d}}{\mathrm{d} t} \int_{a}^{t} f(x) E_{\alpha}\left(\frac{-\alpha}{1-\alpha}(t-x)^{\alpha}\right) \mathrm{d} x, \\
& { }^{\mathrm{ABC}} D_{a+}^{\alpha} f(t)=\frac{B(\alpha)}{1-\alpha} \int_{a}^{t} f^{\prime}(x) E_{\alpha}\left(\frac{-\alpha}{1-\alpha}(t-x)^{\alpha}\right) \mathrm{d} x .
\end{aligned}
$$

In this way we are able to describe a different type of dynamics of nonlocal complex systems. In fact the classical fractional calculus and the one corresponding to the MittagLeffler nonsingular kernel complement each other in the attempt to better describe the hidden aspects of nonlocal dynamical systems. Fractional calculus based on the nonsingular Mittag-Leffler kernel is more easily used from the numerical viewpoint, and this has been studied for example in [31].

We note that the Mittag-Leffler function is already known to be highly useful in fractional calculus [46]. Applications of the new $\mathrm{AB}$ formula have been explored in fields as diverse as chaos theory [9], heat transfer [10], and variational problems [2]. Furthermore, it is natural to address the same questions about the fractional integrator and applications of these new operators in the theory of control and related fractional variational EulerLagrange and Hamilton equations (see [1, 31, 36-38]). Besides, we expect to obtain some new terms in all generalized formulae from the classical fractional calculus, and this aspect will be important for the related applications.

Some basic properties of the new $\mathrm{AB}$ differintegrals have already been proved in several recent papers: for example, the original paper [10] established the formulae for Laplace transforms of $A B$ differintegrals and some Lipschitz-type inequalities; the paper [2] considered integration by parts identities and Euler-Lagrange equations; and the paper [31] established, using Laplace transforms, analogues of the Newton-Leibniz formula for the integral of a derivative. However, much of the ground-level theory of this new model of fractional calculus has not yet been fully developed, and this paper aims to add to this basic theory by establishing new fundamental results in the field. 
The theory of differential inclusions has recently been an object of increasing interest because of its wide applicability in physics, in biology, and in engineering fields. In fact, classical differential equations cannot be used to describe processes which at certain moments change their state rapidly. Therefore, inclusions differential equations seems to be an appropriate model to describe inclusions problems. Several articles have studied the existence of mild solutions and controllability problems for various types of integers (see [19-32, 34] and the references therein).

In this work, using the fractional-time derivative with the nonsingular Mittag-Leffler kernel [10], we obtain the existence and uniqueness results for a variational fractional differential system in a bounded domain for all the values $\alpha \in(0,1)$ in the following form.

Let $\Omega$ be an open and bounded subset of $R^{d}(d \geq 1)$, with sufficiently smooth boundary $\partial \Omega$. Given $\alpha \in(0,1)$, a well-behaved function $f \in L^{2}\left(0, T ; H^{-1}(\Omega)\right)$, and initial data $y_{0} \in$ $H_{0}^{1}(\Omega)$, we seek

$$
y \in L^{2}\left(0, T ; H_{0}^{1}(\Omega)\right) \cap C\left([0, T] ; L^{2}(\Omega)\right) \cap W^{1,2}\left([0, T] ; H^{-1}(\Omega)\right)
$$

such that

$$
\begin{aligned}
& \left.{ }_{0}^{\mathrm{ABC}} D_{t}^{\alpha} y(t)-\Delta y(t)+\partial \phi(y(t)) \ni f(t), \quad \text { a.e. } t \in\right] 0, T[ \\
& y(x, 0)=y_{0}, \quad x \in \Omega, \\
& y(x, t)=0, \quad x \in \Gamma, t \in(0, T),
\end{aligned}
$$

where ${ }_{0}^{\mathrm{ABC}} D_{t}^{\alpha} y(t)$ for $\alpha \in(0,1)$ is the Atangana-Baleanu fractional times derivatives in the Caputo sense which are formally defined in Sect. 2.

In the cases of single and multiple fractional time classical Caputo and RiemannLiouville derivatives, similar problems to (1.1) have been already studied. In [7], Ahmad and Ntouyas study the existence of solutions for fractional differential inclusions with four-point nonlocal Riemann-Liouville type integral boundary conditions. In [16] the classical control theory has been applied to a fractional inclusions problems in a bounded domain of type (1.1)-(1.3), where the classical Riemann-Liouville fractional derivative with respect to time was considered. In [32], Djida et al. study the optimal control of diffusion equation with fractional time derivative with nonlocal and nonsingular MittagLeffler kernel. In [37], Gomez-Aguilar study the space-time fractional diffusion equation using a derivative with nonsingular and regular kernel. In [28, 29], Barbu studies the necessary conditions for distributed non-fractional control problems governed by parabolic variational inequalities. In [34], Friedman studies the optimal control for non-fractional variational inequalities. These equations have been solved numerically by using different approaches (see, e.g., [6,31] and the references therein).

Although for the Caputo fractional derivative there exist a large and rapidly growing number of publications (see $[12,16]$ ) according to authors' knowledge, we would like to emphasize here the existence and uniqueness of solutions for the fractional inclusions problems of type (1.1)-(1.3), when the classical Caputo fractional-time derivative is not considered for $\alpha(1 / 2,1)$.

Furthermore, as far as the optimal control of problem (1.1)-(1.3) is concerned, one can refer to the methods of the Lagrange multiplier technique for the classical Caputo and 
Riemann-Liouville fractional time derivative presented by different authors (see, e.g., [1$19,26]$ and the references therein).

Making use of the Dubovitskii-Milyutin theorem [35], following in [43], Kotarski considered an optimization problem for a parabolic system where the state of the equation was given by scalar case (one equation), and he obtained necessary and sufficient conditions for optimality by applying the classical Dubovitskii-Milyutin theorem [35]. The performance index was more general than the quadratic one and had an integral form. The set representing the constraints on the controls was assumed to have a nonempty interior. This assumption can be easily removed if we apply the generalized version of the Dubovitskii-Milyutin theorem [50] instead of the classical one [35] (as the approximation of the set of controls, the regular tangent cone is used instead of the regular admissible cone).

The optimal control problems and calculus of variation for variational equality with fractional time derivative with nonlocal and nonsingular Mittag-Leffler kernel are studied in many papers (see, for example, $[1,2,10,11,21]$ and the references therein).

Here we study the optimal control problem for variational inequality with fractional time derivative with nonlocal and nonsingular Mittag-Leffler kernel. We are interested in finding the control $u \in L^{2}(Q)$ that minimizes the cost function

$$
I(u)=\int_{Q} F(x, t, y, u) d x d t
$$

subject to problem (1.1)-(1.3), where $F: \Omega \times(0, T) \times \Omega \times \Omega \rightarrow \mathbb{R}^{1}$ satisfies the following conditions:

$\left(A_{1}\right) F(x, t, y, u)$ is continuous with respect to $(x, t, y, u)$;

$\left(A_{2}\right)$ There exist $F_{y}(x, t, y, u), F_{u}(x, t, y, u)$ which are continuous with respect to $(x, t, y, u)$;

$\left(A_{3}\right) F(x, t, y, u)$ is strictly convex with respect to the pair $(y, u)$, i.e., $\forall y^{1}, y^{2}, u^{1}, u^{2} \in \Omega$, $\left(y^{1}, u^{1}\right) \neq\left(y^{2}, u^{2}\right), \varepsilon \in(0,1)$.

$$
F\left(x, t, \varepsilon y^{1}+(1-\varepsilon) y^{2}, \varepsilon u^{1}+(1-\varepsilon) u^{2}\right)<\varepsilon F\left(x, t, y^{1}, u^{1}\right)+(1-\varepsilon) F\left(x, t, y^{2}, u^{2}\right) .
$$

In other words, we are interested in finding the control parameter $u^{0} \in L^{2}(Q)$ such that the functional

$$
I\left(u^{0}\right)=\inf _{v \in L^{2}(Q)} I(v)
$$

holds true.

This manuscript is structured as follows: In Sect. 2 we collect notation, definitions, and preliminary results regarding the solution representation, and we compute the integration by parts involving the Atangana-Baleanu fractional-time derivative. Consequently, the weak formulation of (1.1)-(1.3) is obtained. Section 3 is dedicated to the sketch of proof of the existence and uniqueness of solution of the weak formulation problem. More precisely, we prove Theorem 3.2 as well as some corollaries. Moreover, Sect. 4 is devoted to an optimal control problem for differential inclusion problem (1.1)-(1.3). 


\section{Preliminaries}

In this section, we give some notations on the functional spaces, useful properties on the Mittag-Leffler function and the fractional time derivatives, regularity results for the fractional variational inequalities with nonsingular Mittag-Leffler kernel (1.1)-(1.3).

Let $L^{2}(\Omega)$ be the usual Hilbert space equipped with the scalar product $(\cdot, \cdot)$, and $H^{m}(\Omega)$, $H_{0}^{m}(\Omega)$ denote the usual Sobolev spaces.

We first start by recalling the Mittag-Leffler function $E_{\alpha, \beta}(z)$ (see $[42,47]$ ) which will be used extensively throughout this work and is defined below:

$$
\begin{aligned}
& E_{\alpha}(z)=\sum_{k=0}^{\infty} \frac{z^{k}}{\Gamma(k \alpha+1)}=: E_{\alpha, 1}(z), \quad E_{\alpha, \beta}(z)=\sum_{k=0}^{\infty} \frac{z^{k}}{\Gamma(k \alpha+\beta)}, \\
& E_{\alpha, 1}(z)=E_{\alpha}(z), \quad z \in \mathbb{C}, \mathcal{R}(\alpha)>0,
\end{aligned}
$$

where $\Gamma(\cdot)$ denotes the gamma function defined as

$$
\Gamma(z)=\int_{0}^{\infty} t^{z-1} e^{-t} d t, \quad \mathcal{R}(z)>0 .
$$

The Mittag-Leffler function is a two-parameter family of entire functions of $z$. The exponential function is a particular case of the Mittag-Leffler function, namely

$$
\begin{aligned}
& E_{1,1}(z)=e^{z}, \quad E_{2,1}(z)=\cosh \sqrt{z}, \quad E_{1,2}(z)=\frac{e^{z}-1}{z}, \quad E_{2,2}(z)=\frac{\sinh \sqrt{z}}{\sqrt{z}}, \\
& E_{\alpha, \beta}^{\lambda}(z)=\sum_{k=0}^{\infty} \frac{(\lambda)_{k}}{\Gamma(k \alpha+\beta)} \frac{z^{k}}{n !}, \quad z, \beta, \lambda \in \mathbb{C}, \mathcal{R}(\alpha)>0,
\end{aligned}
$$

where (and throughout this investigation) $(\lambda)_{k}$ denotes the familiar Pochhammer symbol or the shifted factorial. Furthermore, we recall the following lemma from [42].

Lemma 2.1 Let $\alpha, \beta \in \mathbb{C}$ such that $\mathcal{R}(\alpha)>0$ and $\mathcal{R}(\beta)>0$. Then we have that

$$
\left(\frac{d}{d z}\right) E_{\alpha, \beta}(z)=\frac{1}{\alpha}\left[(1+\alpha-\beta) E_{\alpha, \beta}(z)+E_{\alpha, \beta-1}(z)\right], \quad z \in \mathbb{C} .
$$

Let us recall some useful definitions of fractional derivatives in the sense of AtanganaBaleanu [10].

Definition 2.1 ([31, 32]) For a given function $u \in H^{1}(a, T), T>a, \alpha \in(0,1)$, the Atangana-Baleanu fractional derivative (AB derivative) of $u$ of order $\alpha$ in the Caputo sense ${ }_{a}^{\mathrm{ABC}} D_{t}^{\alpha} u(t)$ (where A denotes Atangana, B denotes Baleanu, and $\mathrm{C}$ denotes Caputo type) with base point $a$ is defined at a point $t \in(a, T)$ by

$$
\left.{ }_{a}^{\mathrm{ABC}} D_{t}^{\alpha} u(t)=\frac{B(\alpha)}{1-\alpha} \int_{a}^{t} u^{\prime}(s) E_{\alpha}\left[-\gamma(t-s)^{\alpha}\right] d s \quad \text { (left } \mathrm{ABCD}\right),
$$

where

$$
\gamma=\frac{\alpha}{(1-\alpha)}
$$


$E_{\alpha}(\cdot)$ stands for the Mittag-Leffler function, and with $B(\alpha)$ being a normalization function satisfying

$$
B(\alpha)=(1-\alpha)+\frac{\alpha}{\Gamma(\alpha)}, \quad \text { where } B(0)=B(1)=1,
$$

and in the Riemann-Liouville sense with

$$
{ }_{a}^{\mathrm{ABR}} D_{t}^{\alpha} u(t)=\frac{B(\alpha)}{1-\alpha} \frac{d}{d t} \int_{a}^{t} u(s) E_{\alpha}\left[-\gamma(t-s)^{\alpha}\right] d s \quad \text { (left ABRD). }
$$

For $\alpha=1$ in (2.3) we consider the usual classical derivative $\partial_{t}$.

The associated left $\mathrm{AB}$ fractional integral ${ }_{a}^{\mathrm{AB}} I_{t}^{\alpha} u(t)$ is also defined as

$$
{ }_{a}^{\mathrm{AB}} I_{t}^{\alpha} u(t)=\frac{1-\alpha}{B(\alpha)} u(t)+\frac{\alpha}{B(\alpha) \Gamma(\alpha)} \int_{a}^{t} u(s)(t-s)^{\alpha-1} d s \quad \text { (left ABI). }
$$

Notice that if $\alpha=0$ in (2.4) we recover the initial function and if $\alpha=1$ in (2.4) we consider the usual ordinary integral. Some recent results and properties concerning this operator can be found in [1] and papers therein. In addition, we recall the following definition from [1].

Definition 2.2 For a given function $u \in H^{1}(a, T), T>t>a$, the right Atangana-Baleanu fractional derivative of $u$ of order $\alpha$ in the Caputo sense with base point $T$ is defined at a point $t \in(a, T)$ by

$$
{ }_{T}^{\mathrm{ABC}} D_{t}^{\alpha} g(t)=-\frac{B(\alpha)}{1-\alpha} \int_{t}^{T} g^{\prime}(s) E_{\alpha}\left[-\gamma(s-t)^{\alpha}\right] d s \quad \text { (right ABCD), }
$$

and in the Riemann-Liouville sense with

$$
{ }_{T}^{\mathrm{ABR}} D_{t}^{\alpha} g(t)=-\frac{B(\alpha)}{1-\alpha} \frac{d}{d t} \int_{t}^{T} g(s) E_{\alpha}\left[-\gamma(s-t)^{\alpha}\right] d s \quad \text { (right ABRD). }
$$

The associated right $\mathrm{AB}$ fractional integral ${ }_{a}^{\mathrm{AB}} I_{t}^{\alpha} u(t)$ is also defined as

$$
{ }_{t}^{\mathrm{AB}} I_{T}^{\alpha} u(t)=\frac{1-\alpha}{B(\alpha)} u(t)+\frac{\alpha}{B(\alpha) \Gamma(\alpha)} \int_{t}^{T} u(s)(s-t)^{\alpha-1} d s \quad \text { (right ABI). }
$$

Next we state the following proposition which gives integration by parts (see [1]).

Proposition 2.2 (Integration by parts, see [1]) Let $\alpha>0, p \geq 1, q \geq 1$, and $\frac{1}{p}+\frac{1}{q} \leq 1+\alpha$ $\left(p \neq 1\right.$ and $q \neq 1$ in the case $\left.\frac{1}{p}+\frac{1}{q}=1+\alpha\right)$. Then, for any $\phi(x) \in L^{p}(a, b), \psi(x) \in L^{q}(a, b)$, we have

$$
\begin{aligned}
& \int_{a}^{b} \phi(x)_{a}^{\mathrm{AB}} I_{t}^{\alpha} \psi(x) d x=\int_{a}^{b} \psi(x){ }_{t}^{\mathrm{AB}} I_{b}^{\alpha} \phi(x) d x, \\
& \int_{a}^{b} \phi(x)_{t}^{\mathrm{AB}} I_{b}^{\alpha} \psi(x) d x=\int_{a}^{b} \psi(x)_{a}^{\mathrm{AB}} I_{t}^{\alpha} \phi(x) d x
\end{aligned}
$$


if $\phi(x) \in{ }_{t}^{\mathrm{AB}} I_{b}^{\alpha}\left(L^{p}\right)$ and $\psi(x) \in{ }_{a}^{\mathrm{AB}} I_{t}^{\alpha}\left(L^{q}\right)$, then

$$
\begin{aligned}
& \int_{a}^{b} \phi(x){ }_{a}^{\mathrm{ABR}} D_{t}^{\alpha} \psi(x) d x=\int_{a}^{b} \psi(x){ }_{t}^{\mathrm{ABR}} D_{b}^{\alpha} \phi(x) d x, \\
& \int_{a}^{b} \phi(x){ }_{a}^{\mathrm{ABC}} D_{t}^{\alpha} \psi(x) d x=\int_{a}^{b} \psi(x){ }_{t}^{\mathrm{ABR}} D_{b}^{\alpha} \phi(x) d x+\left.\frac{B(\alpha)}{1-\alpha} \psi(t) E_{\alpha, 1, \frac{-\alpha}{1-\alpha}, b}^{1} \phi(t)\right|_{a} ^{b}, \\
& \int_{a}^{b} \phi(x){ }_{t}^{\mathrm{ABC}} D_{b}^{\alpha} \psi(x) d x=\int_{a}^{b} \psi(x){ }_{a}^{\mathrm{ABR}} D_{t}^{\alpha} \phi(x) d x-\left.\frac{B(\alpha)}{1-\alpha} \psi(t) E_{\alpha, 1, \frac{-\alpha}{1-\alpha}, a}^{1} \phi(t)\right|_{a} ^{b},
\end{aligned}
$$

where the left generalized fractional integral operator

$$
E_{\gamma, \mu, \omega, a}^{\alpha} x(t)=\int_{a}^{t}(t-\tau)^{\mu-1} E_{\gamma, \mu}^{\alpha}\left[\omega(t-\tau)^{\gamma}\right] x(\tau) d \tau, \quad t>a,
$$

and the right generalized fractional integral operator

$$
E_{\gamma, \mu, \omega, b}^{\alpha} x(t)=\int_{t}^{b}(\tau-t)^{\mu-1} E_{\gamma, \mu}^{\alpha}\left[\omega(\tau-t)^{\gamma}\right] x(\tau) d \tau, \quad t<b .
$$

We recall some useful relations of the Laplace transform $(\mathcal{L}[\cdot])$ of the generalized Mittag-Leffler function (see [42, 47]):

$$
\begin{aligned}
& \mathcal{L}\left[t^{\alpha}\right](s)=\frac{\Gamma(\alpha+1)}{s^{\alpha+1}} \quad(\mathcal{R}(\alpha)>-1, \mathcal{R}(s)>0), \\
& \mathcal{L}\left[t^{\beta-1} E_{\alpha, \beta}\left(\lambda t^{\alpha}\right)\right](s)=\frac{s^{\alpha-\beta}}{s^{\alpha}-\lambda} \quad\left(\mathcal{R}(\alpha)>-1, \lambda \in \mathbb{C},\left|\lambda s^{\alpha}\right|<1\right), \\
& \mathcal{L}[f(t) * g(t)](s)=\mathcal{L}[f(t)](s) \cdot \mathcal{L}[g(t)](s) .
\end{aligned}
$$

The following lemma gives estimates of the behavior of the Mittag-Leffler function in the complex plane (see [47]).

Lemma 2.3 Let $\beta$ be an arbitrary real number, $\alpha \in(0,2)$, and $\mu$ is such that $\frac{\alpha \pi}{2}<\mu<$ $\min (\pi, \alpha \pi)$. There exists a positive constant $C>0$ such that

$$
\left|E_{\alpha, \beta}(z)\right| \leq \frac{C}{1+|z|}, \quad \mu \leq|\arg (z)| \leq \pi
$$

Next we state the following proposition which gives the fractional Green's formula that will be used in our analysis.

Proposition 2.4 (Fractional Green's formula, see [31]) Let $0<\alpha \leq 1$. Then, for any $\phi, y \in$ $C^{\infty}(\bar{Q})$, we have

$$
\begin{aligned}
\int_{0}^{T} & \int_{\Omega}\left({ }_{0}^{\mathrm{ABC}} D_{t}^{\alpha} y(x, t)-\Delta y(x, t)\right) \phi(x, t) d x d t \\
= & \int_{0}^{T} \int_{\partial \Omega} y \frac{\partial \phi}{\partial \nu} d \Gamma d t-\int_{0}^{T} \int_{\partial \Omega} \phi \frac{\partial y}{\partial \nu} d \Gamma d t \\
& +\frac{B(\alpha)}{1-\alpha} \int_{\Omega} \phi(x, T) \int_{0}^{T} y(x, t) E_{\alpha, \alpha}\left[-\gamma(T-t)^{\alpha}\right] d t d x
\end{aligned}
$$




$$
\begin{aligned}
& -\frac{B(\alpha)}{1-\alpha} \int_{\Omega} \int_{0}^{T} y(x, 0) E_{\alpha, \alpha}\left[-\gamma t^{\alpha}\right] \phi(x, t) d t d x \\
& +\int_{0}^{T} \int_{\Omega} y(x, t)\left(-{ }_{T}^{\mathrm{ABC}} D_{t}^{\alpha} \phi(x, t)-\Delta \phi(x, t)\right) d x d t .
\end{aligned}
$$

We also introduce the Hilbert space

$$
\mathcal{W}(0, T):=\left\{y: y \in L^{2}\left(0, T ; H_{0}^{1}(\Omega)\right),{ }_{0}^{\mathrm{ABC}} D_{t}^{\alpha} y(t) \in L^{2}\left(0, T ; H^{-1}(\Omega)\right)\right\}
$$

in which a solution of a fractional differential system is contained. The spaces considered in this paper are assumed to be real.

\subsection{Definitions of cones and Lyusternik theorem}

Milyutin-Dubovitskii's method $[49,50]$ arises from the geometric from the Hahn-Banach theorem (theorem about separation of convex sets). It will be shown in the example.

Let us assume that $E$ is a linear topological space, locally convex, $I(x)$ is a functional defined on $E, A_{i}, i=1,2, \ldots, n$, are sets in $E$ with inner points which represent inequality constraints, $B$ is a set in $E$ without inner points representing equality constraints.

We must find some conditions necessary for a local minimum of the functional $I(x)$ on the set $Q=\bigcap_{i=1}^{n} A_{i} \cap B$ or find a point $x_{0} \in E$ so that $I\left(x_{0}\right)=\min _{Q \cup U} I(x)$, where $U$ means certain neighborhood of the point $x_{0}$. We define the set

$$
A_{0}=\left\{x: I(x)<I\left(x_{0}\right)\right\}
$$

Then we formulate the necessary condition of optimality as follows: in the neighborhood of the local minimum point, the intersection of the class of sets (the set on which the functional attains smaller values than $I\left(x_{0}\right)$, and the sets representing constraints) is empty or $\bigcap_{i=0}^{n} A_{i} \cap B=\phi$.

The condition $\bigcap_{i=0}^{n} A_{i} \cap B=\phi$ is also equivalent to the one in which, instead of sets $A_{i}, i=$ $1,2, \ldots, n$, or $B$, there are approximations of $A_{i}, i=1,2, \ldots, n$, and $B$. These approximations are cones with the vertices at a point $\{0\}$.

We shall approximate the inequality constraints by the regular admissible cones $\operatorname{RAC}\left(A_{i}, x_{0}\right), i=1,2, \ldots, n$, the equality constraints by the regular tangent cone $\operatorname{RTC}\left(B, x_{0}\right)$, and for the performance functional, we shall construct the regular improvement cone $\operatorname{RFC}\left(I, x_{0}\right)$.

Then we have the necessary condition of the optimality $I(x)$ on the set $Q=\bigcap_{i=1}^{n} A_{i} \cap B$ that has the form of Euler-Lagrange's equation

$$
\sum_{i=0}^{n+1} f_{i}=0
$$

where $f_{i}(i=0,1, \ldots, n+1)$ are the linear, continuous functionals; all of them are not equal to zero at the same time, and they belong to the adjoint cones

$$
\begin{aligned}
& f_{i} \in\left[\operatorname{RAC}\left(A_{i}, x_{0}\right)\right]^{*}, \quad i=1,2, \ldots, n, \quad f_{n+1} \in\left[\operatorname{RTC}\left(B, x_{0}\right)\right]^{*}, \quad f_{0} \in\left[\operatorname{RFC}\left(I_{i}, x_{0}\right)\right]^{*}, \\
& \left\{f_{i} \in\left[\operatorname{RAC}\left(A_{i}, x_{0}\right)\right]^{*} \Leftrightarrow f_{i}(x) \geq 0 \forall x \in \operatorname{RAC}\left(A_{i}, x_{0}\right), i=1,2, \ldots, n\right\} .
\end{aligned}
$$


For the convex problem, i.e., the problem in which the constraints are convex sets and the performance functional is convex, the Euler-Lagrange equation is the necessary and sufficient condition of optimality, provided that certain additional assumptions are fulfilled (the so-called Slater condition).

At first we recall definitions of conical approximations and cones of the same sense or of the opposite sense. Let $A$ be a set contained in a Banach space $X$ and $F: X \rightarrow \mathbb{R}$ be a given functional.

Definition 2.3 (see $[43,50])$ A set TC $\left(A, x^{0}\right):=\left\{h \in X: \exists \epsilon_{0}>0, \forall \epsilon \in\left(0, \epsilon_{0}\right), \exists r(\epsilon) \in X ; x^{0}+\right.$ $\epsilon h+r(\epsilon) \in A\}$, where $\frac{r(\epsilon)}{\epsilon} \rightarrow 0$ as $\epsilon \rightarrow 0$ is called the tangent cone to the set $A$ at the point $x^{0} \in A$.

Definition 2.4 (see $[43,50]$ ) A set $\mathrm{AC}\left(A, x^{0}\right):=\left\{h \in X: \exists \epsilon_{0}>0, \exists U(h), \forall \epsilon \in\left(0, \epsilon_{0}\right), \forall \bar{h} \in\right.$ $\left.U(h) ; x^{0}+\epsilon \bar{h} \in A\right\}$, where $U(h)$ is a neighborhood of $h$, is called the admissible cone to the set $A$ at the point $x^{0} \in A$.

Definition 2.5 (see $[43,50]$ ) A set $\mathrm{FC}\left(F, x^{0}\right):=\left\{h \in X: \exists \epsilon_{0}>0, \exists U(h), \forall \epsilon \in\left(0, \epsilon_{0}\right), \forall \bar{h} \in\right.$ $\left.U(h) ; F\left(x^{0}+\epsilon \bar{h}\right)<F\left(x^{0}\right)\right\}$, is called the cone of decrease of the functional $F$ at the point $x^{0} \in X$.

Definition 2.6 (see $[43,50])$ A set $\mathrm{NC}\left(F, x^{0}\right):=\left\{h \in X: \exists \epsilon_{0}>0, \exists U(h), \forall \epsilon \in\left(0, \epsilon_{0}\right), \forall \bar{h} \in\right.$ $\left.U(h) ; F\left(x^{0}+\epsilon \bar{h}\right) \leq F\left(x^{0}\right)\right\}$, is called the cone of nonincrease of the functional $F$ at the point $x^{0} \in X$.

All the cones defined above are cones with vertices at the origin. The cones $\operatorname{AC}\left(A, x^{0}\right)$, $\mathrm{FC}\left(F, x^{0}\right)$, and $\mathrm{NC}\left(F, x^{0}\right)$ are open, while the cone $\operatorname{TC}\left(A, x^{0}\right)$ is closed. If $\operatorname{int} A \neq \emptyset$, then $\operatorname{AC}\left(A, x^{0}\right)$ does not exist. Moreover, if $A_{1}, \ldots, A_{n} \in X, x^{0} \in \bigcap_{i=1}^{n} A_{i}$, then

$$
\bigcap_{i=1}^{n} \mathrm{TC}\left(A_{i}, x^{0}\right) \supset \mathrm{TC}\left(\bigcap_{i=1}^{n} A_{i}, x^{0}\right) \text { and } \bigcap_{i=1}^{n} \mathrm{AC}\left(A_{i}, x^{0}\right)=\operatorname{AC}\left(\bigcap_{i=1}^{n} A_{i}, x^{0}\right) \text {. }
$$

If the cones $\mathrm{TC}\left(A, x^{0}\right), \mathrm{AC}\left(A, x^{0}\right), \mathrm{FC}\left(F, x^{0}\right)$, and $\mathrm{NC}\left(F, x^{0}\right)$ are convex, then they are called regular cones and we denote them by $\operatorname{RTC}\left(A, x^{0}\right), \operatorname{RAC}\left(A, x^{0}\right), \operatorname{RFC}\left(F, x^{0}\right)$, and $\operatorname{RNC}\left(F, x^{0}\right)$, respectively.

Let $C_{i}, i=1, \ldots, n$, be a system of cones and $B_{M}$ be a ball with center 0 and radius $M>0$ in the space $X$.

Definition 2.7 (see $[43,50]$ ) The cones $C_{i}, i=1, \ldots, n$, are of the same sense if $\forall M>0$, $\exists M_{1}, \ldots, M_{2}>0$ so that $\forall x \in B_{M} \cap \sum_{i=1}^{n} C_{i}, x=\sum_{i=1}^{n} x_{i}, x_{i} \in C_{i}, i=1, \ldots, n$, we have $x_{i} \in$ $B_{M_{i}} \cap C_{i}, i=1, \ldots, n$ (or equivalently the inequality $\|x\| \leq M$ implies the inequalities $\left\|x_{i}\right\| \leq$ $\left.M_{i}, i=1, \ldots, n\right)$.

Definition 2.8 (see $[43,50]$ ) The cones $C_{i}, i=1, \ldots, n$, are of the opposite sense if $\exists\left(x_{1}, \ldots, x_{n}\right) \neq(0, \ldots, 0), x_{i} \in C_{i}, i=1, \ldots, n$, so that $0=\sum_{i=1}^{n} x_{i}$.

Remark 2.5 (see $[43,50]$ ) From Definitions 2.6 and 2.7 it follows that the set of cones of the same sense is disjoint with the set of cones of the opposite sense. If a certain subsystem of cones is of the opposite sense, then the whole system is also of the opposite sense. 
In finite dimensional spaces only the cones of the two types mentioned above may exist, while in arbitrary infinite dimensional normed spaces the situation is more complicated as shown in the example below.

Example 2.1 (see $[43,50]$ ) In the space $C^{1}[0,1]$ we take the norm $\|x\|:=\sqrt{\int_{0}^{1} x^{2}(t) d t}$ and define the functional $A(x):=\left.\frac{d}{d t} x(t)\right|_{t=\frac{1}{2}}=: r \in \mathbb{R}$. That functional is linear and bounded (in fact, for the sequence $x_{n}(t)=\frac{1}{\sqrt{n}} \sin 2 \pi n t$, we have $\left\|x_{n}\right\|=\frac{1}{\sqrt{2 n}} \rightarrow 0$, while $A\left(x_{n}\right)=$ $\left.2 \pi \sqrt{n} \cos \pi n=2 \pi \sqrt{n}(-1)^{n} \not \rightarrow 0\right)$. Further, we define $C_{1}:=\operatorname{cl}\{(x, r) ; r=A(x)\}, C_{2}:=\{\Theta\} \times \mathbb{R}$ closed and convex cones in $E:=L^{2}(0,1) \times \mathbb{R}$ with the norm $\|(x, r)\|_{E}:=\max \{\|x\|,|r|\}$. The cones $C_{1}$ and $C_{2}$ are not of the same sense. To prove this, we take $v_{1}=\left(x_{1}, r_{1}\right) \in C_{1}$, $v_{2}=\left(\Theta, r_{2}\right) \in C_{2}$, and an arbitrary constant $M>0$.

If

$$
\left\|v_{1}+v_{2}\right\|_{E}=\max \left\{\left\|x_{1}\right\|,\left|r_{1}+r_{2}\right|\right\} \leq M
$$

then the following inequalities

$$
\left\|v_{1}\right\|_{E} \leq M_{1}, \quad\left\|v_{2}\right\|_{E} \leq M_{2}
$$

generally do not hold with some $M_{1}, M_{2}>0$. Actually, since $A$ is an unbounded functional, then there exists $\tilde{M}_{1}>0$ so that $\left|r_{1}\right|=\left|A\left(x_{1}\right)\right|>\tilde{M}_{1}$. Further fix $M<\tilde{M}_{1}$. Then $M \geq \mid r_{1}+$ $r_{2}|\geq| r_{1}|-| r_{2}\left|>\tilde{M}_{1}-\right| r_{2} \mid$ and finally $\left\|v_{2}\right\|_{E}=\tilde{M}_{1}-M=: M_{2}>0$, which contradicts the same sense of the cones $C_{1}$ and $C_{2}$ are also not of the opposite sense.

In $[49,50]$ the conditions, under which a system of cones is of the same sense, are given.

Definition 2.9 (see $[43,50]$ ) Let $K$ be a cone in $X$. The adjoint cone $K^{*}$ of $K$ is defined as

$$
K^{*}:=\left\{f \in X^{*} ; f(x) \geq 0 \forall x \in K\right\}
$$

where $X^{*}$ denotes the dual space of $X$.

Definition 2.10 (see $[43,50]$ ) Let $Q$ be a set in $X, x^{0} \in Q$. A functional $f \in X^{*}$ is said to be a support functional to the set $Q$ at $x^{0}$ if $f(x) \geq f\left(x^{0}\right) \forall x \in Q$.

Lemma 2.6 (Tangent directions, see [35]) The theorem of Lyusternik proved below is a powerful tool for the calculation of tangent directions. Before proceeding to a statement of the theorem, we recall the definition of a differentiable operator. Let $E_{1}, E_{2}$ be Banach spaces, $P(x)$ be an operator (generally nonlinear) with domain in $E_{1}$ and range in $E_{2}$. Then $P(x)$ is said to be differentiable at a point $x_{0} \in E_{1}$ if there exists a continuous linear operator A mapping $E_{1}$ into $E_{2}$ such that, for all $h \in E_{1}$,

$$
P\left(x_{0}+h\right)=P\left(x_{0}\right)+A h+r\left(x_{0}, h\right)
$$

where $\left\|r\left(x_{0}, h\right)\right\|=O(\|h\|)$, The operator $A$ is called the Frèchet derivative of the operator $P(x)$ and often denoted by $A=P^{\prime}\left(x_{0}\right)$. It is clear that if $E_{2}=R^{1}$ (i.e., $P(x)$ is a functional), this definition coincides with the derivative of a functional. The derivative of an operator 
possesses the usual properties of derivatives (rules for differentiation of sums, composite functions, etc.). The derivative of a continuous linear operator coincides with the operator.

Theorem 2.7 (Lyusternik theorem, see [35]) Let $P(x)$ be an operator mapping $E_{1}$ into $E_{2}$, differentiable in a neighborhood of a point $x_{0}, P\left(x_{0}\right)=0$. Let $P^{\prime}(x)$ be continuous in a neighborhood of $x_{0}$, and suppose that $P^{\prime}\left(x_{0}\right)$ maps $E_{1}$ onto $E_{2}$ (i.e., the linear equation $P^{\prime}\left(x_{0}\right) h=b$ has a solution $h$ for any $\left.b \in E_{2}\right)$. Then the set of tangent directions $K$ to the set $Q=\{x: P(x)=0\}$ at the point $x_{0}$ is the subspace $K=\left\{h: P^{\prime}\left(x_{0}\right) h=0\right\}$.

The proof of this theorem (which is by no means trivial) may be found in [35].

\subsection{Generalized Dubovitskii-Milyutin theorem}

Let $X$ be a Banach space, $Q_{k} \subset X$, int $Q_{k} \neq \emptyset, k=1, \ldots, p$, represent inequality constraints, $Q_{k} \subset X$, int $Q_{k}=\emptyset, k=p+1, \ldots, n$, represent equality constraints, $I_{i}:=X \rightarrow \mathcal{R}, i=1, \ldots, s$, are given functionals $I=\left(I_{1}, \ldots, I_{s}\right)^{T}$, i.e., $I: X \rightarrow \mathcal{R}^{s}$ is a vector performance index. We are interested in the following problem (see $[43,50])$.

Problem (P): Find $x^{0} \in Q$ such that

$$
\min _{x \in \mathrm{Q} \cap U\left(x^{0}\right)} I(x)=I\left(x^{0}\right)
$$

where $Q=\bigcap_{k=1}^{n} Q_{k}$ and $U\left(x^{0}\right)$ is some neighborhood of $x^{0}$.

If we define equality constraints in the operator form

$$
Q_{k}:=\left\{x \in X: F_{k}(x)=0\right\},
$$

where $F_{k}: X \rightarrow Y_{k}$ are given operators, $Y_{k}$ are Banach spaces, $k=p+1, \ldots, n$, then we obtain Problem (P1) instead of Problem (P).

Definition 2.11 (see $[43,50]$ ) A point $x^{0} \in X$ is called global (local) optimal for Problem (P) or (P1) if $x^{0} \in Q$ and there is no $x^{0} \neq x \in Q\left(Q \cap U\left(x^{0}\right)\right)$ with $I_{i}(x) \leq I\left(x^{0}\right)$ for $i=1, \ldots, s$, with strict inequality for at least one $i, 1 \leq i \leq s$.

Theorem 2.8 (Generalized Dubovitskii-Milyutin theorem, see [43, 50]) We assume for problem (P) that:

(i) the cones $K_{i}, i=1, \ldots, s, D_{j}, j=1, \ldots, s, C_{k}, k=1, \ldots, p$, are open and convex;

(ii) the cones $C_{k}, k=p+1, \ldots, n$, are convex and closed;

(iii) the cone $\tilde{C}=\bigcap_{k=p+1}^{n} C_{k}$ is contained in the cone tangent to the set $\bigcap_{k=p+1}^{n} Q_{k}$;

(iv) the cones $C_{k}^{*}, k=p+1, \ldots, n$, are either of the same sense or of the opposite sense,

(v) $x^{0} \in Q$ is a local optimum for problem (P),

then the following "s" equations (the so-called Euler-Lagrange equations) must hold:

$$
f_{i}+\sum_{j=1, j \neq i} f_{j}^{(i)}+\sum_{k=1}^{n} \varphi_{k}^{(i)}=0, \quad i=1,2, \ldots, s,
$$

where $f_{i} \in K_{i}^{*}, f_{j}^{(i)} \in D_{j}^{*}, j=1, \ldots, s, j \neq i, \varphi_{k}^{(i)} \in C_{k}^{*}, k=1, \ldots, n$, with not all functionals equal to zero simultaneously. 
Case $s=1$

Problems (P) and (P1) for $s=1$ convert to scalar ones. For them we have the following.

Theorem 2.9 (see $[43,50])$ Let us assume for Problem (P) that:

(i) $s=1$;

(ii) $I: X \rightarrow \mathcal{R}$ is convex and continuous;

(iii) $Q_{k}, k=1, \ldots, n$ are convex;

(iv) there exists $\tilde{x}$ so that $\tilde{x} \in\left(\bigcap_{k=1}^{p}\right.$ int $\left.Q_{k}\right) \cap\left(\bigcap_{k=p+1}^{n}\right.$ int $\left.Q_{k}\right)$;

(v) $\operatorname{RTC}\left(\bigcap_{k=p+1}^{n} Q_{k}, x^{0}\right)=\bigcap_{k=p+1}^{n} \operatorname{RTC}\left(Q_{k}, x^{0}\right)$;

(vi) the cones $\left[\operatorname{RTC}\left(Q_{k}, x^{0}\right)\right]^{*}, k=p+1, \ldots, n$, are either of the same sense or of the opposite sense,

then $x^{0}$ is a global optimum for Problem (P) if and only if the Euler-Lagrange equation

$$
f_{1}+\sum_{k=1}^{n} \varphi_{k}=0
$$

holds, where $f \in\left[\operatorname{RFC}\left(I, x^{0}\right)\right]^{*}, \varphi_{k} \in\left[\operatorname{RAC}\left(Q_{k}, x^{0}\right)\right]^{*}, k=1, \ldots, p$, and $\varphi_{k} \in\left[\operatorname{RAC}\left(Q_{k}, x^{0}\right)\right]^{*}$, $k=p+1, \ldots, n$, and the functionals are not simultaneously equal to zero.

Using Milyutin-Dubovitskii's theorem, we shall derive the necessary conditions of optimality for differential inclusions with Mittag-Leffler kernel.

\section{Fractional problem for differential inclusions with Mittag-Leffler kernel}

For $y_{0} \in H_{0}^{1}(\Omega)$ and $f \in L^{2}\left(0, T ; H^{-1}(\Omega)\right)$, let us consider the fractional variational inequalities: Find $y(t) \in \mathcal{W}(0, T)$ such that

$$
\begin{aligned}
& \left.{ }_{0}^{\mathrm{ABC}} D_{t}^{\alpha} y(t)-\Delta y(t)+\partial \phi(y(t)) \ni f(t), \quad \text { a.e. } t \in\right] 0, T[ \\
& y(x, 0)=y_{0}, \quad x \in \Omega, \\
& y(x, t)=0, \quad x \in \Gamma, t \in(0, T),
\end{aligned}
$$

where ${ }_{0}^{\mathrm{ABC}} D_{t}^{\alpha} y(t)$ is the $\mathrm{AB}$ fractional derivatives of $y:[0, t] \rightarrow H^{-1}(\Omega)$ in the Caputo sense defined by (2.2).

Definition 3.1 In terms of a subgradient mapping $\partial \phi: H_{0}^{1}(\Omega) \rightarrow H^{-1}(\Omega)$, where

$$
\begin{cases}\partial \phi(x)=\left\{g \in H^{-1}(\Omega) ; \phi(x)-\phi(y) \leq(g, x-y), \forall y \in H_{0}^{1}(\Omega)\right\}, & \text { if } x \in D(\phi), \\ \partial \phi(x)=\emptyset, & \text { if } x \notin D(\phi),\end{cases}
$$

where $\phi$ is a lower semicontinuous convex function $\phi: H_{0}^{1}(\Omega) \rightarrow(-\infty,+\infty]$ and

$$
D(\phi)=\left\{x \in H_{0}^{1}(\Omega) ; \phi(x)<\infty\right\}, \quad D(\partial \phi)=\left\{x \in H_{0}^{1}(\Omega) ; \partial \phi(x) \neq \emptyset\right\}
$$

so that $D(\partial \phi) \subset D(\phi)$. 
Inequality (3.1) can be written as follows:

$$
\begin{aligned}
& \left({ }_{0}^{\mathrm{ABC}} D_{t}^{\alpha} y(t)-\Delta y(t), y(t)-z\right)+\phi y(t)-\phi(z) \\
& \quad \leq(f(t), y(t)-z), \quad \text { a.e. } t \in] 0, T\left[, \forall z \in H_{0}^{1}(\Omega) .\right.
\end{aligned}
$$

For this operator $\Delta=\sum_{i=1}^{n} \frac{\partial^{2}}{\partial x_{i}^{2}}$, we define the bilinear form as follows.

Definition 3.2 We define a bilinear form $\pi(y, \psi)$ on $H_{0}^{1}(\Omega)$ by

$$
\pi(y, \psi)=\int_{\Omega} \nabla y(x) \nabla \psi(x) d x
$$

so that we have $\pi(y, \psi)=\pi(\psi, y) \forall y, \psi \in H_{0}^{1}(\Omega)$, and

$$
\pi(y, y)=\|y\|_{H_{0}^{1}(\Omega)}^{2}
$$

Then (3.1) can be written as follows:

$$
\begin{aligned}
& \pi(y(t), y(t)-z)+\left({ }_{0}^{\mathrm{ABC}} D_{t}^{\alpha} y(t), y(t)-z\right)+\phi(y(t))-\phi(z) \\
& \leq(f(t), y(t)-z), \quad \text { a.e. } t \in] 0, T\left[, \forall z \in H_{0}^{1}(\Omega) .\right.
\end{aligned}
$$

In the special case when $\phi=I_{K}$ is the indicator function of some closed convex subset $K$ of $H_{0}^{1}(\Omega)$, i.e.,

$$
\phi(y)=0 \quad \text { if } y \in K, \quad \phi(y)=+\infty \quad \text { if } y \notin K,
$$

the variational inequality (3.1) reduces to

$$
\begin{aligned}
& y(t) \in K \quad \text { for all } t \in[0, T], \\
& \left.\left({ }_{0}^{\mathrm{ABC}} D_{t}^{\alpha} y(t)-\Delta y(t), y(t)-z\right) \leq(f(t), y(t)-z), \quad \text { a.e. } t \in\right] 0, T[, \forall z \in K,
\end{aligned}
$$

which can be written as

$$
\left.\pi(y(t), y(t)-z)+\left({ }_{0}^{\mathrm{ABC}} D_{t}^{\alpha} y(t), y(t)-z\right) \leq(f(t), y(t)-z), \quad \text { a.e. } t \in\right] 0, T[, \forall z \in K .
$$

Interpretation of problem (3.9). Let $\psi$ be in $\mathcal{D}(\Omega \times] 0, \infty[)$; if we formally take $y=z \pm \psi$ in (3.9) (this can be justified by a construction), we obtain

$$
\left({ }_{0}^{\mathrm{ABC}} D_{t}^{\alpha} y(t), \psi\right)_{L^{2}(\Omega)}+\pi(y(t), \psi)_{L^{2}(\Omega)}=(f(t), \psi)_{L^{2}(\Omega)}, \quad \forall \psi \in H_{0}^{1}(\Omega),
$$

and since $\psi$ depends only on $x$, we observe that $\left.{ }^{\mathrm{ABC}} D_{t}^{\alpha} y(t), \psi\right)_{L^{2}(\Omega)}={ }_{0}^{\mathrm{ABC}} D_{t}^{\alpha}(y(t), \psi)_{L^{2}(\Omega)}$. Therefore, the fractional Cauchy variational problem involving the Atangana-Baleanu fractional derivative with nonsingular Mittag-Leffler kernel is as follows: Find $y(t)$, a function of ]0, $T$ [, with values in $H_{0}^{1}(\Omega)$ such that

$$
\begin{aligned}
& { }_{0}^{\mathrm{ABC}} D_{t}^{\alpha}(y(t), \psi)_{L^{2}(\Omega)}+\pi(y(t), \psi)_{L^{2}(\Omega)}=(f(t), \psi)_{L^{2}(\Omega)}, \quad \forall \psi \in H_{0}^{1}(\Omega), \\
& y(x, 0)=y_{0}, \quad x \in \Omega .
\end{aligned}
$$


Proposition 3.1 Let $f_{i} \in L^{2}(Q)$ such that the Atangana-Baleanu fractional derivative exists. Then the solution of differential equations

$$
\begin{aligned}
& \left.{ }_{0}^{\mathrm{ABC}} D_{t}^{\alpha} y_{i}(t)+\lambda_{i} y_{i}(t)=f_{i}(t), \quad \text { a.e. } t \in\right] 0, T[. \\
& y_{i}(x, 0)=y_{i 0}, \quad x \in \Omega
\end{aligned}
$$

is given by

$$
\begin{aligned}
y_{i}(x)= & \zeta_{i} E_{\alpha}\left[-\gamma_{i} t^{\alpha}\right] y_{i 0}+\frac{\gamma_{i} \zeta_{i}}{\gamma}\left[{ }_{0}^{\mathrm{AB}} I_{t}^{\alpha} f_{i}(t)-\frac{1-\alpha}{B(\alpha)} f_{i}(t)\right] \\
& +\frac{\alpha \zeta_{i}^{2}}{B(\alpha)} \int_{0}^{t}(t-s)^{\alpha-1} E_{\alpha, \alpha}\left[-\gamma_{i}(t-s)^{\alpha}\right] f_{i}(s) d s,
\end{aligned}
$$

where the constants $\gamma_{i}, \zeta_{i}$, and $k_{i}$ are given by

$$
\gamma_{i}=\frac{\alpha \lambda_{i}}{\left(B(\alpha)+(1-\alpha) \lambda_{i}\right)}, \quad \zeta_{i}=\frac{B(\alpha)}{\left(B(\alpha)+(1-\alpha) \lambda_{i}\right)}
$$

Iff $\in H^{1}(Q)$, then

$$
\begin{aligned}
y_{i}(x)= & {\left[\zeta_{i} y_{i}(0)-\frac{\alpha \zeta_{i}^{2}}{\gamma_{i} B(\alpha)} f_{i}(0)\right] E_{\alpha}\left[-\gamma_{i} t^{\alpha}\right]+\left[\frac{\gamma \alpha \zeta_{i}^{2}-\gamma_{i}^{2} \zeta_{i}(1-\alpha)}{\gamma_{i} \gamma B(\alpha)} f_{i}(t)\right] } \\
& +\frac{\gamma_{i} \zeta_{i}}{\gamma}{ }_{0}{ }_{0} I_{t}^{\alpha} f_{i}(t)-\frac{\alpha \zeta_{i}^{2}}{B(\alpha)} \int_{0}^{t} f_{i}^{\prime}(s) E_{\alpha}\left[-\gamma_{i}(t-s)^{\alpha}\right] d s .
\end{aligned}
$$

Proof We apply the Laplace transform on both sides of equation (3.12)

$$
\mathcal{L}\left[{ }_{0}^{\mathrm{ABC}} D_{t}^{\alpha} y_{i}(t)\right]+\lambda_{i} \mathcal{L}\left[y_{i}(t)\right]=\mathcal{L}\left[f_{i}(t)\right]
$$

Note that we can rewrite the derivative as the product of convolution of two functions. Thus, it follows that

$$
\begin{aligned}
& \mathcal{L}\left[{ }_{0}^{\mathrm{ABC}} D_{t}^{\alpha} y_{i}(t)\right]=\frac{B(\alpha)}{1-\alpha} \mathcal{L}\left[E_{\alpha}\left[-\gamma t^{\alpha}\right] * y_{i}^{\prime}(t)\right]=\frac{B(\alpha)}{1-\alpha} \mathcal{L}\left[E_{\alpha}\left[-\gamma t^{\alpha}\right]\right] \mathcal{L}\left[y_{i}^{\prime}(t)\right], \\
& \mathcal{L}\left[{ }_{0}^{\mathrm{ABC}} D_{t}^{\alpha} y_{i}(t)\right]=\frac{B(\alpha)}{1-\alpha} \mathcal{L}\left[E_{\alpha}\left[-\gamma t^{\alpha}\right]\right]\left[s \mathcal{L}\left[y_{i}(t)\right]-y_{i}(0)\right] .
\end{aligned}
$$

Equation (3.16) becomes

$$
\left(\frac{B(\alpha)}{1-\alpha} S \mathcal{L}\left[E_{\alpha}\left[-\gamma t^{\alpha}\right]\right]+\lambda_{i}\right) \mathcal{L}\left[y_{i}(t)\right]=\frac{B(\alpha)}{1-\alpha} \mathcal{L}\left[E_{\alpha}\left[-\gamma t^{\alpha}\right]\right] y_{i}(0)+\mathcal{L}\left[f_{i}(t)\right]
$$

Using relation (2.15) with $\beta=1$, we have

$$
\mathcal{L}\left[E_{\alpha}\left[-\gamma t^{\alpha}\right]=\frac{s^{\alpha-1}}{s^{\alpha}+\gamma}\right.
$$


It follows that

$$
\begin{aligned}
\frac{B(\alpha)}{1-\alpha} S \mathcal{L}\left[E_{\alpha}\left[-\gamma t^{\alpha}\right]\right]+\lambda_{i} & =\frac{s^{\alpha}(B(\alpha)+\lambda(1-\alpha))+\lambda \alpha}{(1-\alpha)\left(s^{\alpha}+\gamma\right)} \\
& =\frac{s^{\alpha}+\gamma_{i}}{s^{\alpha}+\gamma} \cdot \frac{B(\alpha)}{\zeta_{i}(1-\alpha)}
\end{aligned}
$$

where the constants $\gamma_{i}$ and $\zeta_{i}$ are given by

$$
\gamma_{i}=\frac{\alpha \lambda_{i}}{\left(B(\alpha)+(1-\alpha) \lambda_{i}\right)}, \quad \zeta_{i}=\frac{B(\alpha)}{\left(B(\alpha)+(1-\alpha) \lambda_{i}\right)} .
$$

Then equation (3.17) becomes

$$
\mathcal{L}\left[y_{i}(t)\right]=\zeta_{i} \frac{s^{\alpha-1}}{s^{\alpha}+\gamma_{i}} y_{i}(0)+\frac{\zeta_{i}(1-\alpha)}{B(\alpha)} \cdot \frac{s^{\alpha}+\gamma}{s^{\alpha}+\gamma_{i}} \mathcal{L}\left[f_{i}(t)\right] .
$$

Using (2.15) and applying the inverse Laplace transform, it holds

$$
\begin{aligned}
& \mathcal{L}^{-1}\left[\frac{s^{\alpha-1}}{s^{\alpha}+\gamma_{i}}\right]=E_{\alpha}\left[-\gamma_{i} t^{\alpha}\right], \\
& \mathcal{L}^{-1}\left[\frac{s^{\alpha}}{s^{\alpha}+\gamma_{i}}\right]=t^{-1} E_{\alpha, 0}\left[-\gamma_{i} t^{\alpha}\right]=\frac{\gamma_{i} t^{\alpha-1}}{\Gamma(\alpha)}-\gamma_{i} t^{\alpha-1} E_{\alpha, \alpha}\left[-\gamma_{i} t^{\alpha}\right], \\
& \mathcal{L}^{-1}\left[\frac{\gamma}{s^{\alpha}+\gamma_{i}}\right]=\gamma t^{\alpha-1} E_{\alpha, \alpha}\left[-\gamma_{i} t^{\alpha}\right] .
\end{aligned}
$$

By applying the inverse Laplace transform on both sides of (3.18), we observe

$$
\begin{aligned}
y_{i}(t) & =\zeta_{i} E_{\alpha}\left[-\gamma_{i} t^{\alpha}\right] y_{i}(0)+\left[\frac{\gamma_{i} \zeta_{i}(1-\alpha)}{B(\alpha) \Gamma(\alpha)} t^{\alpha-1}+\left(\gamma-\gamma_{i}\right) \frac{\zeta_{i}(1-\alpha)}{B(\alpha)} t^{\alpha-1} E_{\alpha, \alpha}\left[-\gamma_{i} t^{\alpha}\right]\right] * f(t) \\
& =\zeta_{i} E_{\alpha}\left[-\gamma_{i} t^{\alpha}\right] y_{i}(0)+\left[\frac{\gamma_{i} \zeta_{i}(1-\alpha)}{B(\alpha) \Gamma(\alpha)} t^{\alpha-1} * f(t)+\frac{\alpha \zeta_{i}^{2}}{B(\alpha)} t^{\alpha-1} E_{\alpha, \alpha}\left[-\gamma_{i} t^{\alpha}\right] * f(t)\right] \\
& =\zeta_{i} E_{\alpha}\left[-\gamma_{i} t^{\alpha}\right] y_{i}(0)+\left[\frac{\gamma_{i} \zeta_{i}(1-\alpha)}{B(\alpha)}{ }_{0}^{\mathrm{RL}} I_{t}^{\alpha} f(t)+\frac{\alpha \zeta_{i}^{2}}{B(\alpha)} t^{\alpha-1} E_{\alpha, \alpha}\left[-\gamma_{i} t^{\alpha}\right] * f(t)\right]
\end{aligned}
$$

To complete the proof of (3.14), we use (2.9).

If $f \in H^{1}(Q)$, then by integration by parts, we get that

$$
\int_{0}^{t}(t-s)^{\alpha-1} E_{\alpha, \alpha}\left[-\gamma_{i}(t-s)^{\alpha}\right] f_{i}(s) d s=f_{i}(t)-f_{i}(0) E_{\alpha}\left[-\gamma_{i} t^{\alpha}\right]-\int_{0}^{t} f_{i}^{\prime}(s) E_{\alpha}\left[-\gamma_{i}(t-s)^{\alpha}\right] d s .
$$

This completes the proof of (3.15), we use (2.9).

Therefore, we have the following existence theorem. 
Theorem 3.2 Let $\alpha \in(0,1)$. Assume $f \in L^{2}\left((0, T) ; L^{2}(\Omega)\right), y_{0} \in L^{2}(\Omega)$. Then problem (3.10) has a unique solution $y \in L^{2}\left(0, T ; H_{0}^{1}(\Omega)\right) \cap \mathcal{C}\left(0, T ; L^{2}(\Omega)\right) \cap \mathcal{W}(0, T)$ given by

$$
\begin{aligned}
y_{i}(x, t)= & \zeta_{i} E_{\alpha}\left[-\gamma_{i} t^{\alpha}\right] y_{i 0}+\frac{\gamma_{i} \zeta_{i}}{\gamma}\left[{ }_{0}^{\mathrm{AB}} I_{t}^{\alpha} f_{i}(t)-\frac{1-\alpha}{B(\alpha)} f_{i}(t)\right] \\
& +\frac{\alpha \zeta_{i}^{2}}{B(\alpha)} \int_{0}^{t}(t-s)^{\alpha-1} E_{\alpha, \alpha}\left[-\gamma_{i}(t-s)^{\alpha}\right] f_{i}(s) d s, \\
y(x, t)= & \sum_{i=1}^{+\infty} y_{i}(x, t) \omega_{i},
\end{aligned}
$$

where $\left\{\omega_{i}\right\}_{i=1}^{\infty}$ are the orthogonal eigenfunctions corresponding to the eigenvalues $\left\{\lambda_{i}\right\}_{i=1}^{\infty}$ of the operator $(-\Delta)$. Moreover, $y$ satisfies the bounded conditions

$$
\|y\|_{L^{2}\left(0, T ; H_{0}^{1}(\Omega)\right)}+\|y\|_{\mathcal{C}\left(0, T ; L^{2}(\Omega)\right)} \leq \Lambda\left(\left\|y_{0}\right\|_{H_{0}^{1}(\Omega)}+\|f\|_{L^{2}\left((0, T) ; L^{2}(\Omega)\right)}\right)
$$

where

$$
\begin{aligned}
\Lambda= & \max \left\{\sqrt{3} C, \frac{2 \sqrt{3} \gamma T^{\alpha-\frac{1}{2}}}{(2 \alpha+1) \Gamma(\alpha)}\left[1+\Gamma(\alpha)^{2} C^{2}\right]^{\frac{1}{2}},\right. \\
& \sqrt{\frac{3 \mathrm{TC}^{2}}{\lambda_{1}(1-\alpha)^{2}}}, \sqrt{\left.3\left[\frac{\gamma^{2} T^{2 \alpha}}{2 \alpha \lambda_{1} \Gamma(\alpha)^{2}}+\frac{\gamma^{2} C^{2} T^{2 \alpha}}{2 \alpha^{3} \lambda_{1}^{3}}\right]\right\}} .
\end{aligned}
$$

Proof The proof is divided into two steps. In the first step, assuming the existence of a solution $y$, we obtain an explicit formula for $y$ in the form of a series obtained by spectral decomposition of the spaces $H_{0}^{1}(\Omega)$ and $L^{2}(\Omega)$. In particular, this formula proves the uniqueness of the solution. In a second step, we show that this series converges in spaces $L^{2}\left(0, T ; H_{0}^{1}(\Omega)\right)$ and $\mathcal{C}\left(0, T ; L^{2}(\Omega)\right)$.

Step 1: Suppose that $y \in L^{2}\left(0, T ; H_{0}^{1}(\Omega)\right) \cap \mathcal{C}\left(0, T ; L^{2}(\Omega)\right)$. We use the decomposition method in a Hilbert basis of $L^{2}(\Omega)$. For this purpose, it is very convenient to choose the orthogonal basis $\left\{\omega_{i}\right\}_{i=1}^{\infty}$ of $L^{2}(\Omega)$ composed of eigenfunctions of $-\Delta$ (with zero Dirichlet condition). The eigenfunctions $\left\{\omega_{i}\right\}_{i=1}^{\infty}$ correspond to the strictly positive real eigenvalues $\left\{\lambda_{i}\right\}_{i=1}^{\infty}$, i.e.,

$$
w_{i} \in H_{0}^{1}(\Omega), \quad \pi\left(\omega_{i}, v\right)=\left(-\Delta \omega_{i}, v\right)=\lambda_{i}\left(\omega_{i}, v\right)_{L^{2}(\Omega)}, \quad \forall v \in H_{0}^{1}(\Omega) .
$$

We define

$$
y_{i}(t)=\left\langle y(t), w_{i}\right\rangle_{L^{2}(\Omega)}, \quad y_{i}^{0}=\left\langle y^{0}, w_{i}\right\rangle_{L^{2}(\Omega)}, \quad f_{i}(t)=\left\langle f(t), w_{i}\right\rangle_{L^{2}(\Omega)} .
$$

Since $y \in L^{2}\left(0, T ; H_{0}^{1}(\Omega)\right) \cap \mathcal{C}\left(0, T ; L^{2}(\Omega)\right)$ and $f \in L^{2}\left(0, T ; H_{0}^{1}(\Omega)\right)$, we deduce that $y_{i}(t) \in$ $\mathcal{C}(0, T)$ and $f_{i}(t) \in L^{2}(0, T)$. Using the fact that $\left\{\omega_{i}\right\}_{i=1}^{\infty}$ is an orthogonal basis of $L^{2}(\Omega)$, we have

$$
y(t)=\sum_{i=1}^{+\infty} y_{i}(t) \omega_{i} .
$$


By choosing $\psi=\omega_{i}$ in (3.10), we obtain

$$
\begin{aligned}
& { }_{0}^{\mathrm{ABC}} D_{t}^{\alpha} y_{i}(t)+\lambda_{i} y_{i}(t)=f_{i}(t), \quad \text { a.e. } t \in[0, T], \\
& y_{i}(0)=y_{i}^{0}, \quad x \in \Omega .
\end{aligned}
$$

Using Proposition 3.1, the solution of this fractional differential equation takes the form

$$
\begin{aligned}
y_{i}(t)= & \zeta_{i} E_{\alpha}\left[-\gamma_{i} t^{\alpha}\right] y_{i}^{0}+\frac{\gamma_{i} \zeta_{i}}{\gamma}\left[{ }_{0}^{\mathrm{AB}} I_{t}^{\alpha} f_{i}(t)-\frac{1-\alpha}{B(\alpha)} f_{i}(t)\right] \\
& +\frac{\alpha \zeta_{i}^{2}}{B(\alpha)} \int_{0}^{t}(t-s)^{\alpha-1} E_{\alpha, \alpha}\left[-\gamma_{i}(t-s)^{\alpha}\right] f_{i}(s) d s .
\end{aligned}
$$

This ends step one and proves the uniqueness of the solution.

Step 2: We will prove that the series $y(t)=\sum_{i=1}^{+\infty} y_{i}(t) \omega_{i}$ converges in $y \in L^{2}\left(0, T ; H_{0}^{1}(\Omega)\right) \cap$ $\mathcal{C}\left(0, T ; L^{2}(\Omega)\right)$. Consider the partial sum of order $k$ of this series

$$
y^{k}(t)=\sum_{i=1}^{k} y_{i}(t) \omega_{i}
$$

Clearly, $y^{k}(t)$ belongs to $\mathcal{C}\left(0, T ; L^{2}(\Omega)\right)$ since each $y_{i}(t)$ is continuous. Let us show that the sequence $y^{k}(t)$ is a Cauchy sequence in the space $\mathcal{C}\left(0, T ; L^{2}(\Omega)\right)$. Let $p$ and $q$ be two natural numbers such that $p>q$, and using the orthogonality of the eigenfunctions, we have

$$
\begin{aligned}
\left\|y^{p}(t)-y^{q}(t)\right\|_{L^{2}(\Omega)}^{2} & =\sum_{i=q+1}^{p} y_{i}(t)^{2} \\
& \leq \sum_{i=q+1}^{p}\left(A_{1}+A_{2}+A_{3}\right)^{2} \\
& \leq 3 \sum_{i=q+1}^{p}\left(A_{1}^{2}+A_{2}^{2}+A_{3}^{2}\right),
\end{aligned}
$$

where

$$
\begin{aligned}
& A_{1}=\zeta_{i} E_{\alpha}\left[-\gamma_{i} t^{\alpha}\right] y_{i}^{0} \\
& A_{2}=\frac{\gamma_{i} \zeta_{i}}{\gamma}\left[{ }_{0}^{\mathrm{AB}} I_{t}^{\alpha} f_{i}(t)-\frac{1-\alpha}{B(\alpha)} f_{i}(t)\right] \\
& A_{3}=\frac{\alpha \zeta_{i}^{2}}{B(\alpha)} \int_{0}^{t}(t-s)^{\alpha-1} E_{\alpha, \alpha}\left[-\gamma_{i}(t-s)^{\alpha}\right] f_{i}(s) d s .
\end{aligned}
$$

The objective is to estimate each term $A_{1}, A_{2}$, and $A_{3}$. Using Lemma 2.3,

$$
A_{1}^{2}=\zeta_{i}^{2}\left(E_{\alpha}\left[-\gamma_{i} t^{\alpha}\right]\right)^{2}\left|y_{i}^{0}\right|^{2} \leq \frac{C^{2} \zeta_{i}^{2}}{1+\left|\gamma_{i} t^{\alpha}\right|}\left|y_{i}^{0}\right|^{2} \leq C^{2} \zeta_{i}^{2}\left|y_{i}^{0}\right|^{2} \leq C^{2}\left|y_{i}^{0}\right|^{2}
$$




$$
\begin{aligned}
A_{2}^{2} & =\frac{\left(\gamma_{i} \zeta_{i}\right)^{2}}{\gamma^{2}}\left[{ }_{0}^{\mathrm{AB}} I_{t}^{\alpha} f_{i}(t)-\frac{1-\alpha}{B(\alpha)} f_{i}(t)\right]^{2}=\frac{\gamma_{i}^{2} \zeta_{i}^{2}(1-\alpha)^{2}}{B(\alpha)^{2}}\left({ }_{0}^{\mathrm{RL}} I_{t}^{\alpha} f(t)\right)^{2} \\
& \left.\leq \frac{\alpha^{2}}{B(\alpha)^{2}}{ }_{0}^{\mathrm{RL}} I_{t}^{\alpha} f_{i}(t)\right)^{2} \leq \gamma^{2}\left({ }_{0}^{\mathrm{RL}} I_{t}^{\alpha} f_{i}(t)\right)^{2} \leq \frac{\gamma^{2}}{\Gamma(\alpha)^{2}}\left(\int_{0}^{t} f_{i}(s)(t-s)^{\alpha-1} d s\right)^{2} \\
& \leq \frac{\gamma^{2} t^{2 \alpha-1}}{\Gamma(\alpha)^{2}} \int_{0}^{T} f_{i}(s)^{2} d s, \\
A_{3}^{2} & =\frac{\alpha^{2} \zeta_{i}^{4}}{B(\alpha)^{2}}\left(\int_{0}^{t}(t-s)^{\alpha-1} E_{\alpha, \alpha}\left[-\gamma_{i}(t-s)^{\alpha}\right] f_{i}(s) d s\right)^{2} \\
& \leq \gamma^{2}\left(\int_{0}^{t}(t-s)^{\alpha-1} E_{\alpha, \alpha}\left[-\gamma_{i}(t-s)^{\alpha}\right] f_{i}(s) d s\right)^{2} \\
& \leq \gamma^{2} t \int_{0}^{T}(t-s)^{2(\alpha-1)} E_{\alpha, \alpha}\left[-\gamma_{i}(t-s)^{\alpha}\right]^{2} f_{i}(s)^{2} d s \\
& \leq \gamma^{2} t^{2 \alpha-1} \int_{0}^{T} E_{\alpha, \alpha}\left[-\gamma_{i}(t-s)^{\alpha}\right]^{2} f_{i}(s)^{2} d s \\
& \leq \gamma^{2} t^{2 \alpha-1} \int_{0}^{T} \frac{C^{2}}{1+\left|\gamma_{i}(t-s)^{\alpha}\right|^{2}} f_{i}(s)^{2} d s \leq \gamma^{2} C^{2} t^{2 \alpha-1} \int_{0}^{T} f_{i}(s)^{2} d s .
\end{aligned}
$$

Therefore, by taking the square root and the supremum, we have

$$
\begin{aligned}
\sup _{t \in[0, T]}\left\|y^{p}(t)-y^{q}(t)\right\|_{L^{2}(\Omega)} \leq & \sqrt{3} C\left(\sum_{i=q+1}^{p}\left|y_{i}^{0}\right|^{2}\right)^{\frac{1}{2}} \\
& +\frac{\sqrt{3} \gamma t^{\alpha-\frac{1}{2}}}{\Gamma(\alpha)}\left[1+\Gamma(\alpha)^{2} C^{2}\right]^{\frac{1}{2}}\left(\sum_{i=q+1}^{p} \int_{0}^{T} f_{i}(s)^{2} d s\right)^{\frac{1}{2}} .
\end{aligned}
$$

By integrating over $t$ and dividing by $T$, we get

$$
\begin{aligned}
\sup _{t \in[0, T]}\left\|y^{p}(t)-y^{q}(t)\right\|_{L^{2}(\Omega)} \leq & \sqrt{3} C\left(\sum_{i=q+1}^{p}\left|y_{i}^{0}\right|^{2}\right)^{\frac{1}{2}} \\
& +\frac{2 \sqrt{3} \gamma T^{\alpha-\frac{1}{2}}}{(2 \alpha+1) \Gamma(\alpha)}\left[1+\Gamma(\alpha)^{2} C^{2}\right]^{\frac{1}{2}}\left(\sum_{i=q+1}^{p} \int_{0}^{T} f_{i}(s)^{2} d s\right)^{\frac{1}{2}} .
\end{aligned}
$$

As $y^{0} \in L^{2}(\Omega)$ and $f \in L^{2}\left(0, T ; L^{2}(\Omega)\right)$, we have

$$
\left\|y^{0}\right\|_{L^{2}(\Omega)}=\left(\sum_{i=1}^{\infty}\left|y_{i}^{0}\right|^{2}\right)^{\frac{1}{2}}<\infty, \quad\|f\|_{L^{2}\left(0, T ; L^{2}(\Omega)\right)}=\left(\sum_{i=1}^{\infty} \int_{0}^{T} f_{i}(s)^{2} d s\right)^{\frac{1}{2}}<\infty
$$

We deduce that the sequence $y^{k}$ satisfies

$$
\lim _{p, q \rightarrow \infty} \sup _{t \in[0, T]}\left\|y^{p}(t)-y^{q}(t)\right\|_{L^{2}(\Omega)}=0
$$

i.e., the sequence $y^{k}$ is Cauchy in $\mathcal{C}\left(0, T ; L^{2}(\Omega)\right)$. 
Let us show that the sequence $y^{k}$ is also Cauchy in $L^{2}\left(0, T ; H_{0}^{1}(\Omega)\right)$. For $p>q$, we have

$$
\begin{aligned}
\left\|y^{p}(t)-y^{q}(t)\right\|_{H_{0}^{1}(\Omega)}^{2} & =\pi\left(y^{p}(t)-y^{q}(t), y^{p}(t)-y^{q}(t)\right)=\sum_{i=q+1}^{p} \lambda_{i} y_{i}(t)^{2} \\
& \leq 3 \sum_{i=q+1}^{p} \lambda_{i}\left(A_{1}^{2}+A_{2}^{2}+A_{3}^{2}\right),
\end{aligned}
$$

where

$$
\begin{aligned}
\lambda_{i} A_{1}^{2} & =\lambda_{i} \zeta_{i}^{2}\left(E_{\alpha}\left[-\gamma_{i} t^{\alpha}\right]\right)^{2}\left|y_{i}^{0}\right|^{2} \leq \frac{\lambda_{i} C^{2} \zeta_{i}^{2}}{1+\left|\gamma_{i} t^{\alpha}\right|}\left|y_{i}^{0}\right|^{2} \leq \frac{C^{2} B(\alpha)^{2}}{\lambda_{1}(1-\alpha)^{2}}\left|y_{i}^{0}\right|^{2} \leq \frac{C^{2}}{\lambda_{1}(1-\alpha)^{2}}\left|y_{i}^{0}\right|^{2}, \\
\lambda_{i} A_{2}^{2} & =\lambda_{i} \frac{\gamma_{i}^{2} \zeta_{i}^{2}(1-\alpha)^{2}}{B(\alpha)^{2}}\left({ }_{0}^{\mathrm{RL}} I_{t}^{\alpha} f(t)\right)^{2} \leq \frac{\gamma^{2}}{\lambda_{1}}\left({ }_{0}^{\mathrm{RL}} I_{t}^{\alpha} f_{i}(t)\right)^{2} \\
& \leq \frac{\gamma^{2}}{\lambda_{1} \Gamma(\alpha)^{2}}\left(\int_{0}^{t} f_{i}(s)(t-s)^{\alpha-1} d s\right)^{2} \leq \frac{\gamma^{2} t^{2 \alpha-1}}{\lambda_{1} \Gamma(\alpha)^{2}} \int_{0}^{T} f_{i}(s)^{2} d s \\
\lambda_{i} A_{3}^{2}= & \lambda_{i} \frac{\alpha^{2} \zeta_{i}^{4}}{B(\alpha)^{2}}\left(\int_{0}^{t}(t-s)^{\alpha-1} E_{\alpha, \alpha}\left[-\gamma_{i}(t-s)^{\alpha}\right] f_{i}(s) d s\right)^{2} \\
& \leq \frac{\gamma^{2} t^{2 \alpha-1}}{\alpha^{2} \lambda_{1}^{3}} \int_{0}^{T} E_{\alpha, \alpha}\left[-\gamma_{i}(t-s)^{\alpha}\right]^{2} f_{i}(s)^{2} d s \leq \frac{\gamma^{2} t^{2 \alpha-1}}{\alpha^{2} \lambda_{1}^{3}} \int_{0}^{T} \frac{C^{2}}{1+\left|\gamma_{i}(t-s)^{\alpha}\right|^{2}} f_{i}(s)^{2} d s \\
& \leq \frac{\gamma^{2} C^{2} t^{2 \alpha-1}}{\alpha^{2} \lambda_{1}^{3}} \int_{0}^{T} f_{i}(s)^{2} d s, \\
\left\|y^{p}-y^{q}\right\|_{L^{2}\left(0, T, H_{0}^{1}(\Omega)\right)}^{2}=\int_{0}^{T}\left\|y^{p}(t)-y^{q}(t)\right\|_{H_{0}^{1}(\Omega)}^{2} d t & \\
\leq & \frac{3 \mathrm{TC} C^{2}}{\lambda_{1}(1-\alpha)^{2}} \sum_{i=q+1}^{p}\left|y_{i}^{0}\right|^{2} \\
& +3\left[\frac{\gamma^{2} T^{2 \alpha}}{2 \alpha \lambda_{1} \Gamma(\alpha)^{2}}+\frac{\gamma^{2} C^{2} T^{2 \alpha}}{2 \alpha^{3} \lambda_{1}^{3}}\right] \sum_{i=q+1}^{p} \int_{0}^{T} f_{i}(s)^{2} d s
\end{aligned}
$$

which implies that the sequence $y^{k}$ satisfies

$$
\lim _{p, q \rightarrow \infty}\left\|y^{p}-y^{q}\right\|_{L^{2}\left(0, T, H_{0}^{1}(\Omega)\right)}=0
$$

i.e., the sequence $y^{k}$ is Cauchy in $L^{2}\left(0, T, H_{0}^{1}(\Omega)\right)$.

Furthermore, using the estimates obtained previously of the proof, we have

$$
\begin{aligned}
\sup _{t \in[0, T]}\|y(t)\|_{L^{2}(\Omega)} \leq & \sqrt{3} C\left\|y^{0}\right\|_{L^{2}(\Omega)} \\
& +\frac{2 \sqrt{3} \gamma T^{\alpha-\frac{1}{2}}}{(2 \alpha+1) \Gamma(\alpha)}\left[1+\Gamma(\alpha)^{2} C^{2}\right]^{\frac{1}{2}}\|f\|_{L^{2}\left(0, T ; H_{0}^{1}(\Omega)\right)}, \\
\|y\|_{L^{2}\left(0, T, H_{0}^{1}(\Omega)\right)} \leq & \sqrt{\frac{3 T C^{2}}{\lambda_{1}(1-\alpha)^{2}}\left\|y^{0}\right\|_{L^{2}(\Omega)}} \\
& +\sqrt{3\left[\frac{\gamma^{2} T^{2 \alpha}}{2 \alpha \lambda_{1} \Gamma(\alpha)^{2}}+\frac{\gamma^{2} C^{2} T^{2 \alpha}}{2 \alpha^{3} \lambda_{1}^{3}}\right]}\|f\|_{L^{2}\left(0, T ; H_{0}^{1}(\Omega)\right)}^{2}
\end{aligned}
$$


setting

$$
\begin{aligned}
\Lambda= & \max \left\{\sqrt{3} C, \frac{2 \sqrt{3} \gamma T^{\alpha-\frac{1}{2}}}{(2 \alpha+1) \Gamma(\alpha)}\left[1+\Gamma(\alpha)^{2} C^{2}\right]^{\frac{1}{2}},\right. \\
& \sqrt{\frac{3 \mathrm{TC}^{2}}{\lambda_{1}(1-\alpha)^{2}}}, \sqrt{3\left[\frac{\gamma^{2} T^{2 \alpha}}{2 \alpha \lambda_{1} \Gamma(\alpha)^{2}}+\frac{\gamma^{2} C^{2} T^{2 \alpha}}{2 \alpha^{3} \lambda_{1}^{3}}\right]} .
\end{aligned}
$$

\section{Optimality conditions for fractional differential inclusions}

For a control $u \in L^{2}(Q)$, the state $y(u)$ of the system is given by the fractional differential inclusions

$$
\begin{aligned}
& { }_{0}^{\mathrm{ABC}} D_{t}^{\alpha} y(t)-\Delta y(t)+\partial \phi(y) \ni f+v, \quad t \in[0, T], \\
& y(0)=y_{0}, \quad x \in \Omega, \\
& y(x, t)=0, \quad x \in \Gamma, t \in(0, T) .
\end{aligned}
$$

The performance functional is given by

$$
I(y, u)=\int_{Q} F(x, t, y, u) d x d t \rightarrow \min ,
$$

where $F: \Omega \times(0, T) \times \Omega \times \Omega \rightarrow \mathbb{R}^{1}$ satisfies conditions $\left(A_{1}\right)-\left(A_{3}\right)$.

We assume the following constraints on controls:

$u \in \mathcal{U}_{\text {ad }}$ is a closed, convex subset in the space $\mathcal{U}=L^{2}(Q)$,

with nonempty interior in the space $\mathcal{U}$.

Control problem: We want to minimize $I$ over $U_{\text {ad }}$, i.e., find the optimal control $v$ such that

$$
I\left(u^{0}\right)=\inf _{u \in U_{\mathrm{ad}}} I(u)
$$

Under the given considerations, we have the following theorem.

Theorem 4.1 The solution of the optimization problem (4.1)-(4.5) exists and is unique under assumptions $\left(A_{1}\right)-\left(A_{3}\right)$. The necessary and sufficient conditions of optimality are characterized by the following system of partial differential equations and inequalities.

State equations

$$
\begin{aligned}
& { }_{0}^{\mathrm{ABC}} D_{t}^{\alpha} y^{0}(t)-\Delta y^{0}(t)+\partial \phi\left(y^{0}\right) \ni f+u^{0}, \quad t \in[0, T], \\
& y^{0}(0)=y_{0}, \quad x \in \Omega, \\
& y^{0}(x, t)=0, \quad x \in \Gamma, t \in(0, T) .
\end{aligned}
$$

Adjoint equations

$$
{ }_{0}^{\mathrm{ABC}} D_{t}^{\alpha} p(u)-\Delta p(u)=F_{y}, \quad \text { in } Q,
$$




$$
\begin{aligned}
& p(u)=0, \quad \text { on } \Sigma, \\
& p(x, T ; u)=0, \quad \text { in } \Omega,
\end{aligned}
$$

Maximum condition

$$
\int_{Q}\left(p(u)+F_{u}\right)\left(u-u^{0}\right) d x d t \geq 0 \quad \forall v \in U_{\mathrm{ad}}
$$

where the superscript 0 denotes the optimal element, $p(u)$ is the adjoint state, and $F_{u}$ are the Frèchet derivatives of $F$ with respect to $u$ at the point $(y, v)$.

Proof According to the generalized Dubovitskii-Milyutin theorem [35], we approximate the set representing the inequality constraints by a regular admissible cone, the equality constraints by a regular tangent cone, and the performance functional by a regular improvement cone.

Equality constraint. The set $Q_{1}$ representing the equality constraint has the form

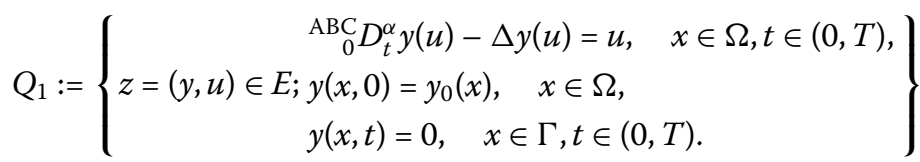

We construct the regular tangent cone of the set $Q_{1}$ using the Lyusternik theorem (Theorem 9.1 [35]). For this purpose, we define the operator $P(y, u)$ in the form

$$
P(y, u):=\left({ }_{0}^{\mathrm{ABC}} D_{t}^{\alpha} y(u)-\Delta y(u)-u, y(x, 0)-y_{0},\left.y(x, t)\right|_{x \in \Gamma}\right) .
$$

The operator $P(y, u)$ is the mapping from the space

$$
\mathcal{W}:=L^{2}\left(0, T, H_{0}^{1}(\Omega)\right) \times L^{2}(Q)
$$

into the space

$$
\mathcal{Z}:=L^{2}\left(0, T ; H_{0}^{-1}(\Omega)\right) \times L^{2}(\Omega) \times L^{2}\left(0, T ; H_{0}^{1}(\Omega)\right)
$$

The Frèchet differential of the operator $P(y, u)$ can be written in the following form:

$$
\begin{aligned}
P^{\prime}\left(y^{0}, u^{0}\right)(\tilde{y}, \tilde{u}) & =\left({ }_{0}^{\mathrm{ABC}} D_{t}^{\alpha} \tilde{y}(u)-\Delta \tilde{y}(u)-u, \tilde{y}(x, 0),\left.\tilde{y}(x, t)\right|_{x \in \Gamma}\right) \\
& =\left\{\begin{array}{c}
{ }_{0}^{\mathrm{ABC}} D_{t}^{\alpha} \tilde{y}(u)-\Delta \tilde{y}(u)=\tilde{u}, \quad x \in \Omega, t \in(0, T) \\
\bar{z} \in E ; \tilde{y}(x, 0)=0, \quad x \in \Omega, \\
\tilde{y}(x, t)=0, \quad x \in \Gamma, t \in(0, T)
\end{array}\right\} .
\end{aligned}
$$

Really, ${ }_{0}^{\mathrm{ABC}} D_{t}^{\alpha}$ [Theorem 2.8 [35]) and $\Delta$ are linear and bounded mappings. Then we can prove that $P^{\prime}\left(y^{0}, u^{0}\right)$ is the operator one-to-one from the space $\mathcal{W}$ onto $\mathcal{Z}$.

Considering that the assumptions of the Lyusternik theorem are fulfilled, we can write the regular tangent cone for the set $Q_{1}$ at the point $\left(y^{0}, u^{0}\right)$ in the form

$$
\operatorname{RTC}\left(Q_{1},\left(y^{0}, u^{0}\right)\right)=\left\{(\tilde{y}, \tilde{u}) \in E ; P^{\prime}\left(y^{0}, u^{0}\right)(\tilde{y}, \tilde{u})=0\right\} .
$$


It is easy to notice that it is a subspace. Therefore, using Theorem 10.1 [35]), we know the form of the functional belonging to the adjoint cone:

$$
f_{1}(\tilde{y}, \tilde{u})=0, \quad \forall(\tilde{y}, \tilde{u}) \in \operatorname{RTC}\left(Q_{1},\left(y^{0}, u^{0}\right)\right) .
$$

Control constraints. The set $Q_{2}=Y \times U_{\text {ad }}$ representing the inequality constraints is closed and convex with nonempty interior in the space $E$ and has the form

$$
Q_{2}:=\left\{z=(y, u) \in E: y \in Y, u \in U_{\mathrm{ad}}\right\}
$$

Using Theorem 10.5 [35], we find the functional belonging to the adjoint regular admissible cone, i.e.,

$$
f_{2}(\tilde{y}, \tilde{u}) \in\left[\operatorname{RTC}\left(Q_{2},\left(y^{0}, u^{0}\right)\right)\right]^{*}
$$

We note that the functional $f_{2}(\tilde{y}, \tilde{u})$ can be expressed as follows:

$$
f_{2}(\tilde{y}, \tilde{u})=f_{2}^{1}\left(\tilde{y}_{i}\right)+f_{2}^{2}(\tilde{u})
$$

where $f_{2}^{1}(\tilde{y})=0 \forall(\tilde{y}) \in Y$ (Theorem 10.1 [35]), $f_{2}^{2}\left(\tilde{u}_{i}\right)$ is the support functional to the set $\mathcal{U}_{\mathrm{ad}}$ at the point $u^{0}$ (Theorem $10.5[35]$ ).

Performance Functional. Using Theorem 7.5 [35], we find the regular cone of decrease

$$
\operatorname{RFC}\left(I, z^{0}\right)=\left\{\tilde{z} \in E ; I^{\prime}\left(z^{0}\right) \tilde{z}<0\right\}
$$

where $I^{\prime}\left(z^{0}\right) \tilde{z}$ is the Frèchet differential of the performance functional.

With assumptions $\left(A_{1}\right),\left(A_{2}\right)$, this differential exists and can be written as follows:

$$
I^{\prime}\left(z^{0}\right) \tilde{z}=\int_{Q}\left(F_{y} \tilde{y}+F_{u} \tilde{u}\right) d x d t
$$

On the basis of Theorem 10.2 [35], if $\operatorname{RFC}\left(I, z^{0}\right) \neq \emptyset$, then the adjoint cone consists of the elements of the form:

$$
f_{3}(\tilde{z})=-\lambda_{0} \int_{Q}\left(F_{y} \tilde{y}+F_{u} \tilde{u}\right) d x d t
$$

where $\lambda_{0} \geq 0$.

Euler-Lagrange equation. The Euler-Lagrange equation for our optimization problem has the form

$$
\sum_{j=1}^{3} f_{j}(\tilde{y}, \tilde{u})=0
$$

Let $p$ be the solution of (4.9)-(4.11) for $u^{0}, y^{0}$, and denote by $\tilde{y}$ the solution of

$$
P^{\prime}\left(y^{0}, u^{0}\right)(\tilde{y}, \tilde{u})=0
$$


for any fixed $\tilde{u}$. Next, taking into account (4.17),(4.18), and (4.20), we can express (4.22) in the form

$$
\left.f_{2}^{2}(\tilde{u})=\lambda_{0} \int_{Q}\left(F_{y} \tilde{y}+F_{u} \tilde{u}\right) d x d t \quad \tilde{\forall} \tilde{y}, \tilde{u}\right) \in \operatorname{RTC}\left(Q_{1},\left(y^{0}, u^{0}\right)\right)
$$

We transform the component with $\tilde{y}$ of the right-hand side of (4.23) introducing the adjoint variable $p$ with the equation [Eq. (4.9)] and using formulas (4.10),(4.15), and (4.16). We obtain

$$
\begin{aligned}
\lambda_{0} \int_{Q} F_{y} \tilde{y}(x, t) d x d t= & \lambda_{0} \int_{0}^{T} \int_{\Omega}\left({ }_{0}^{\mathrm{ABC}} D_{t}^{\alpha} p(u)-\Delta p(u)\right) \tilde{y}(x, t) d x d t \\
= & \lambda_{0} \int_{0}^{T} \int_{\partial \Omega} p(u) \frac{\partial \tilde{y}}{\partial \nu} d \Gamma d t-\lambda_{0} \int_{0}^{T} \int_{\partial \Omega} \tilde{y} \frac{\partial p}{\partial \nu} d \Gamma d t \\
& -\lambda_{0} \frac{B(\alpha)}{1-\alpha} \int_{\Omega} \int_{0}^{T} p(x, 0) E_{\alpha, \alpha}\left[-\gamma t^{\alpha}\right] \tilde{y}(x, t) d t d x \\
& +\lambda_{0} \int_{0}^{T} \int_{\Omega} p(x, t)\left(-{ }^{\mathrm{ABC}}{ }_{T}^{\alpha} D_{t}^{\alpha} \tilde{y}(x, t)-\Delta \tilde{y}(x, t)\right) d x d t \\
= & \lambda_{0} \int_{Q} p \tilde{u} d x d t .
\end{aligned}
$$

Substituting (4.24) into (4.23), we obtain

$$
f_{2}^{2}(\tilde{u})=\lambda_{0} \int_{Q}\left(p+F_{u}\right) \tilde{u} d x d t, \quad \forall \tilde{u} \in \mathcal{U}_{\mathrm{ad}}
$$

A number $\lambda_{0}$ in (4.25) cannot be equal to zero, because in this case all functionals in the Euler-Lagrange equation would be zero, which is impossible according to the Dubovitskii-Milyutin theorem.

Using the definition of the support functional and dividing both sides of the obtained inequalities by $\lambda_{0}$, we finally get

$$
\int_{Q}\left(p+F_{u}\right)\left(u-u^{0}\right) d x d t \geq 0, \quad \forall u \in U_{\mathrm{ad}}
$$

If $\operatorname{RFC}\left(I,\left(y^{0}, u^{0}\right)\right)=\emptyset$, then optimality conditions (4.6)-(4.12) are fulfilled with equality in the maximum condition (4.12).

In order to prove sufficiency of the derived conditions of optimality, we use the fact that the constraints are convex, the performance functional is continuous and convex, and the Slater condition is satisfied [Theorem 15.2 [35]]. Really, there exists a point $(\tilde{y}, \tilde{u}) \in$ int $Q_{2}$ such that $(\tilde{y}, \tilde{u}) \in Q_{1}$. This fact follows immediately from the existence of a nonempty interior in the set $Q_{2}$. The uniqueness of the pair $\left(y^{0}, u^{0}\right)$ follows from the strict convexity of the performance functional (4.4) (assumption $\left(A_{3}\right)$ ). This last remark completes the proof.

Example 4.1 Let $n \in N^{*}$ and $\Omega$ be a bounded open subset of $R^{n}$ with boundary $\partial \Omega$ of class $C^{2}$. For a time $T>0$, we consider the fractional diffusion inequality with AtanganaBaleanu fractional derivative in the Caputo sense (4.1)-(4.3) but we will minimize the cost 
function

$$
J(v)=\left\|y(v)-z_{d}\right\|_{L^{2}(Q)}^{2}+N\|v\|_{L^{2}(Q)}^{2}, \quad z_{d} \in L^{2}(Q), N>0
$$

subject to system (4.1)-(4.3), and the optimal control $v$ will be characterized by system (4.1)-(4.3) with the adjoint system

$$
\begin{aligned}
& { }_{t}^{\mathrm{ABC}} D_{T}^{\alpha} p(t)-\Delta p(t)=y-z_{d}, \quad t \in[0, T], \\
& p(x, t)=0, \quad x \in \Omega, t \in(0, T), \\
& p(x, T)=0, \quad x \in \Gamma,
\end{aligned}
$$

and with the optimality condition

$$
v=-\frac{p}{N} \quad \text { in } Q .
$$

Example 4.2 We can also consider the fractional diffusion inequality with AtanganaBaleanu fractional derivative in the Riemann-Liouville sense:

$$
\begin{aligned}
& { }_{0}^{\mathrm{ABR}} D_{t}^{\alpha} y(t)-\Delta y(t)+\partial \phi(y) \ni f+v, \quad t \in[0, T], \\
& { }_{0} I_{T}^{1-\beta(t)} y\left(0^{+}\right)=y_{0}, \quad x \in \Omega, \\
& y(x, t)=0, \quad x \in \Gamma, t \in(0, T),
\end{aligned}
$$

where ${ }_{0} I_{T}^{1-\beta(t)} y\left(0^{+}\right)=\lim _{t \rightarrow 0^{+}} I_{T}^{1-\beta(t)} y(t)$, the control $v$ belongs to $L^{2}(Q)$. We can minimize the cost function (4.27) subject to system (4.32)-(4.34) and the optimal control $v$ will be characterized by system (4.32)-(4.34) with the adjoint system (4.28)-(4.30) and with the optimality condition (4.31).

\section{Conclusions}

The main purpose of this paper was to study the fractional optimal control problems FOCP for variational differential inclusion involving the AB (Atangana-Baleanu) fractional derivative with Mittag-Leffler nonsingular kernel. The existence and uniqueness of solution were proved by using the spectral theory of $(-\Delta)$, and the control problem has also been studied. We have shown in the first step that the existence of solution was obtained for all values of the fractional parameter $\alpha \in(0,1)$, contrary to the Caputo and Riemann fractional-time derivative where the existence and uniqueness results were obtained for $\alpha \in(1 / 2,1)$ with $y_{0}=0$. This reveals one particularity of the fractional-time derivative with the nonsingular Mittag-Leffler function. Moreover, we have also shown that one can approach the state $y(v)$ of (1.1)-(1.3) by a desired state $z_{d}$ by controlling $v$ and compute the control $u^{0}$ using the algorithm given by the optimality system and following the method of Lagrange. We have used the Dubovitskii-Milyutin theorem and applied the fractional integration by parts in order to derive the necessary optimality conditions for this problem. Also controllability of fractional differential inclusions in Banach spaces involving the $\mathrm{AB}$ (Atangana-Baleanu) fractional derivative with Mittag-Leffler nonsingular kernel has been investigated. We also studied the same problem by considering the Atangana-Baleanu fractional time derivative in the Riemann-Liouville sense. Our further work will be on the study of controllability for fractional stochastic differential inclusions. 


\section{Acknowledgements}

The authors thank the anonymous reviewers for their comments.

\section{Funding}

The authors thank Taibah University for the research funding.

\section{Competing interests}

The authors declare that they have no competing interests.

\section{Authors' contributions}

GMB carried out the study of the optimality conditions for fractional differential inclusions (Sect. 4), participated in the writing of the introduction and preliminaries, and drafted the manuscript. AH carried out the study of the existence and uniqueness of the solution of the fractional differential inclusions problem (Sect. 3), participated in the writing of the introduction and preliminaries, and helped to draft the manuscript. All authors read and approved the final manuscript.

\section{Publisher's Note}

Springer Nature remains neutral with regard to jurisdictional claims in published maps and institutional affiliations.

\section{Received: 6 March 2018 Accepted: 8 July 2018 Published online: 27 July 2018}

\section{References}

1. Abdeljawad, T., Baleanu, D.: Discrete fractional differences with nonsingular discrete Mittag-Leffler kernels. Adv. Differ. Equ. 2016, 232 (2016)

2. Abdeljawad, T., Baleanu, D.: Integration by parts and its applications of a new nonlocal fractional derivative with Mittag-Leffler nonsingular kernel. J. Nonlinear Sci. Appl. 10, 1098-1107 (2017)

3. Agarwal, R.P., Baghli, S., Benchohra, M.: Controllability for semilinear functional and neutral functional evolution equations with infinite delay in Freéchet spaces. Appl. Math. Optim. 60, 253-274 (2009)

4. Agrawal, O.P.: Formulation of Euler-Lagrange equations for fractional variational problems. J. Math. Anal. Appl. 272, 368-379 (2002)

5. Agrawal, O.P.: A general formulation and solution scheme for fractional optimal control problems. Nonlinear Dyn. 38, 323-337 (2004)

6. Agrawal, O.P., Baleanu, D.A.: Hamiltonian formulation and direct numerical scheme for fractional optimal control problems. J. Vib. Control 13(9-10), 1269-1281 (2007)

7. Ahmad, B., Ntouyas, S.K.: Existence of solutions for fractional differential inclusions with four-point nonlocal Riemann-Liouville type integral boundary conditions. Filomat 27(6), 1027-1036 (2013)

8. Al-Salti, N., Karimov, E., Sadarangani, K.: On a differential equation with Caputo-Fabrizio fractional derivative of order $1<\beta \leq 2$ and application to mass-spring-damper system. Prog. Fract. Differ. Appl. 2(4), 257-263 (2016)

9. Alkahtani, B.S.T.: Chua's circuit model with Atangana-Baleanu derivative with fractional order. Chaos Solitons Fractals 89, 547-551 (2016)

10. Atangana, A., Baleanu, D.: New fractional derivatives with non-local and non-singular kernel: theory and application to heat transfer model. Therm. Sci. 20(2), 763-769 (2016)

11. Aydogan, S.M., Baleanu, D., Mousalou, A., Rezapour, S.: On approximate solutions for two higher-order Caputo-Fabrizio fractional integro-differential equations. Adv. Differ. Equ. 2017, 221 (2017). https://doi.org/10.1186/s13662-017-1258-3

12. Bahaa, G.M.: Fractional optimal control problem for differential system with control constraints. Filomat 30(8), 2177-2189 (2016)

13. Bahaa, G.M.: Fractional optimal control problem for infinite order system with control constraints. Adv. Differ. Equ. 2016, $250(2016)$

14. Bahaa, G.M.: Fractional optimal control problem for differential system with delay argument. Adv. Differ. Equ. 2017, 69 (2017)

15. Bahaa, G.M.: Fractional optimal control problem for variable-order differential systems. Fract. Calc. Appl. Anal. 20(6), 1447-1470 (2017)

16. Bahaa, G.M.: Fractional optimal control problem for variational inequalities with control constraints. IMA J. Math. Control Inf. 35(1), 107-122 (2018)

17. Bahaa, G.M., Tang, Q.: Optimality conditions for fractional diffusion equations with weak Caputo derivatives and variational formulation. J. Fract. Calc. Appl. 9(1), 100-119 (2018)

18. Bahaa, G.M., Tang, Q.: Optimal control problem for coupled time-fractional evolution systems with control constraints. J. Differ. Equ. Dyn. Syst. (2017). https://doi.org/10.1007/s12591-017-0403-5

19. Baleanu, D., Agrawal, O.M.P.: Fractional Hamilton formalism within Caputo's derivative. Czechoslov. J. Phys. 56(10/11), 1087-1092 (2000)

20. Baleanu, D., Avkar, T.: Lagrangian with linear velocities within Riemann-Liouville fractional derivatives. Nuovo Cimento B 119, 73-79 (2004)

21. Baleanu, D., Fernandez, A.: On some new properties of fractional derivatives with Mittag-Leffler kernel. Commun. Nonlinear Sci. Numer. Simul. 59, 444-462 (2018)

22. Baleanu, D., Hedayati, V., Rezapour, S., Al Qurashi, M.M.: On two fractional differential inclusions. SpringerPlus 5, 882 (2016). https://doi.org/10.1186/s40064-016-2564-z

23. Baleanu, D., Jajarmi, A., Hajipour, M.: A new formulation of the fractional optimal control problems involving Mittag-Leffler non-singular kernel. J. Optim. Theory Appl. 175(3), 718-737 (2017)

24. Baleanu, D., Mousalou, A., Rezapour, S.: A new method for investigating approximate solutions of some fractional integro-differential equations involving the Caputo-Fabrizio derivative. Adv. Differ. Equ. 2017, 51 (2017). https://doi.org/10.1186/s13662-017-1088-3 
25. Baleanu, D., Mousalou, A., Rezapour, S.: On the existence of solutions for some infinite coefficient-symmetric Caputo-Fabrizio fractional integro-differential equations. Bound. Value Probl. 2017, 145 (2017). https://doi.org/10.1186/s13661-017-0867-9

26. Baleanu, D., Muslih, S.I.: Lagrangian formulation on classical fields within Riemann-Liouville fractional derivatives. Phys. Scr. 72(2-3), 119-121 (2005)

27. Baleanu, D., Rezapour, S., Mohammadi, H.: Some existence results on nonlinear fractional differential equations. Philos. Trans. R. Soc. Lond. A 371, 20120144 (2013)

28. Barbu, V.: Necessary conditions for distributed control problems governed by parabolic variational inequalities. SIAM J. Control Optim. 19, 64-86 (1981)

29. Barbu, V.: Optimal Control of Variational Inequalities. Lecture Notes in Mathematics, vol. 100. Pitman, London (1984)

30. Caputo, M., Fabrizio, M.: A new definition of fractional derivative without singular kernel. Prog. Fract. Differ. Appl. 1(2), 73-85 (2015)

31. Djida, J.D., Atangana, A., Area, I.: Numerical computation of a fractional derivative with non-local and non-singular kernel. Math. Model. Nat. Phenom. 12(3), 4-13 (2017)

32. Djida, J.D., Mophou, G.M., Area, I.: Optimal control of diffusion equation with fractional time derivative with nonlocal and nonsingular Mittag-Leffler kernel. arXiv preprint (2017). arXiv:1711.09070

33. Fernandez, A., Baleanu, D.: The mean value theorem and Taylor's theorem for fractional derivatives with Mittag-Leffler kernel. Adv. Differ. Equ. 201886 (2018)

34. Friedman, A.: Optimal control for variational inequalities. SIAM J. Control Optim. 24(3), 439-451 (1986)

35. Girsanov, I.V.: Lectures on Mathematical Theory of Extremum Problems. Springer, New York (1972)

36. Gomez-Aguilar, J.F.: Irving-Mullineux oscillator via fractional derivatives with Mittag-Leffler kernel. Chaos Solitons Fractals 95(35), 179-186 (2017)

37. Gomez-Aguilar, J.F.: Space-time fractional diffusion equation using a derivative with nonsingular and regular kernel. Phys. Lett. A 465, 562-572 (2017)

38. Gomez-Aguilar, J.F., Atangana, A., Morales-Delgado, J.F.: Electrical circuits RC, LC, and RL described by Atangana-Baleanu fractional derivatives. Int. J. Circuit Theory Appl. 45(11), 1514-1533 (2017). https://doi.org/10.1002/cta.2348

39. Hristov, J.: Transient heat diffusion with a non-singular fading memory. Therm. Sci. 20(2), 757-762 (2016)

40. Jajarmi, A., Hajipour, M., Mohammadzadeh, E., Baleanu, D.: A new approach for the nonlinear fractional optimal control problems with external persistent disturbances. J. Franklin Inst. 335(9), 3938-3967 (2018)

41. Kilbas, A.A., Saigo, M., Saxena, K.: Generalized Mittag-Leffler function and generalized fractional calculus operators. Integral Transforms Spec. Funct. 15(1), 31-49 (2004)

42. Kilbas, A.A., Srivastava, H.M., Trujillo, J.J.: Theory and Applications of Fractional Differential Equations, North-Holland Mathematics Studies, vol. 204. Elsevier, Amsterdam (2006)

43. Kotarski, W.: Some Problems of Optimal and Pareto Optimal Control for Distributed Parameter Systems. Reports of Silesian University, vol. 1668, pp. 1-93. Katowice, Poland (1997)

44. Kumar, D., Singh, J., Baleanu, D.: A new analysis of the Fornberg-Whitham equation pertaining to a fractional derivative with Mittag-Leffler-type kernel. Eur. Phys. J. Plus 133(2), 70 (2018)

45. Kumar, D., Singh, J., Baleanu, D., Sushila: Analysis of regularized long-wave equation associated with a new fractional operator with Mittag-Leffler type kernel. Physica A 492, 155-167 (2018)

46. Mathai, A.M., Haubold, H.J.: Special Functions for Applied Scientists. Springer, New York (2008)

47. Podlubny, I.: Fractional Differential Equations: An Introduction to Fractional Derivatives, Fractional Differential Equations, to Methods of Their Solution and Some of Their Applications, vol. 198. Academic Press, San Diego (1998)

48. Sheikh, N.A., Ali, F., Saqib, M., et al.: Comparison and analysis of the Atangana-Baleanu and Caputo-Fabrizio fractiona derivatives for generalized Casson fluid model with heat generation and chemical reaction. Results Phys. 7, 789-800 (2017)

49. Walczak, S.: On some control problems. Folia Math. 1, 187-196 (1984)

50. Walczak, S.: On some properties of cones in normed spaces and their application to investigating extremal problems J. Optim. Theory Appl. 42(2), 561-582 (1984)

51. Wu, G.-C., Baleanu, D., Huang, L.-L.: Novel Mittag-Leffler stability of linear fractional delay difference equations with impulse. Appl. Math. Lett. 82, 71-78 (2018)

52. Yang, X.J., Gao, F., Machado, J.A., et al.: A new fractional derivative involving the normalized sinc function without singular kernel (2017) arXiv:1701.05590

\section{Submit your manuscript to a SpringerOpen ${ }^{\circ}$ journal and benefit from:}

- Convenient online submission

- Rigorous peer review

- Open access: articles freely available online

- High visibility within the field

- Retaining the copyright to your article

Submit your next manuscript at $>$ springeropen.com 